Portland State University

PDXScholar

Dissertations and Theses

Dissertations and Theses

Winter 3-25-2015

\title{
Vortex Identification in the Wake of a Wind Turbine Array
}

Aleksandr Sergeyevich Aseyev

Portland State University

Follow this and additional works at: https://pdxscholar.library.pdx.edu/open_access_etds

Part of the Energy Systems Commons, and the Power and Energy Commons Let us know how access to this document benefits you.

\section{Recommended Citation}

Aseyev, Aleksandr Sergeyevich, "Vortex Identification in the Wake of a Wind Turbine Array" (2015).

Dissertations and Theses. Paper 2217.

https://doi.org/10.15760/etd.2214

This Thesis is brought to you for free and open access. It has been accepted for inclusion in Dissertations and Theses by an authorized administrator of PDXScholar. Please contact us if we can make this document more accessible: pdxscholar@pdx.edu. 
Vortex Identification in the Wake of a Wind Turbine Array

by

Aleksandr Sergeyevich Aseyev

A thesis submitted in partial fulfillment of the requirements for the degree of

\author{
Master of Science \\ in \\ Mechanical Engineering
}

Thesis Committee:

Raúl Bayoán Cal, Chair

Gerald Recktenwald

Mark Weislogel

Portland State University

2015 


\begin{abstract}
Vortex identification techniques are used to analyze the flow structure in a $4 \times 3$ array of scale model wind turbines. Q-criterion, $\Delta$-criterion, and $\lambda_{2}$-criterion are applied to Particle Image Velocimetry data gathered fore and aft of the last row centerline turbine. Q-criterion and $\lambda_{2}$-criterion provide a clear indication of regions where vortical activity exists while the $\Delta$-criterion does not. Galilean decomposition, Reynolds decomposition, vorticity, and swirling strength are used to further understand the location and behavior of the vortices. The techniques identify and display the high magnitude vortices in high shear zones resulting from the blade tips. Using Galilean and Reynolds decomposition, swirling motions are shown enveloping vortex regions in agreement with the identification criteria. The Galilean decompositions are $20 \%$ and $50 \%$ of a convective velocity of $7 \mathrm{~m} / \mathrm{s}$. As the vortices convect downstream, these vortices weaken in magnitude to approximately $25 \%$ of those present in the near wake. A high level of vortex activity is visualized as a result of the top tip of the wind turbine blade; the location where the highest vertical entrainment commences.
\end{abstract}




\section{Acknowledgements}

The author expresses gratitude to Raúl Bayoán Cal for his guidance and support throughout the project.

Nicholas Hamilton is recognized for his diligence in conducting the experiment and addressing any miscellaneous questions.

Alina Aseyev is applauded for her extraordinary care and patience. 


\section{Contents}

1 Introduction 1

1.1 Wind Energy . . . . . . . . . . . . . . . . . . 1

1.2 Vortex Identification Methods . . . . . . . . . . . . 6

2 Experimental Setup $\quad 19$

3 Analysis Methodology 23

3.1 Two-Dimensional Truncation . . . . . . . . . . . . . . . . . 23

3.2 Method of Calculation . . . . . . . . . . . . . . 25

3.3 Frame Selection . . . . . . . . . . . . . . . . 26

4 Results and Discussion $\quad 27$

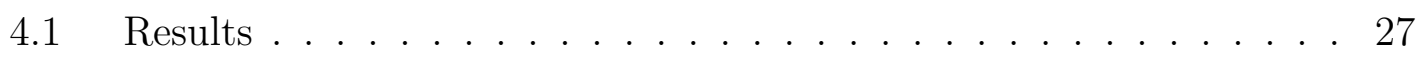

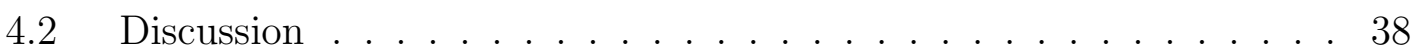

5 Conclusions $\quad 41$

$\begin{array}{ll}\text { Bibliography } & 44\end{array}$

$\begin{array}{ll}\text { Appendices } & 48\end{array}$ 
A Vortex Identification Matlab Codes $\quad 48$

A.1 Vorticity $(\omega) \ldots \ldots \ldots . \ldots \ldots$

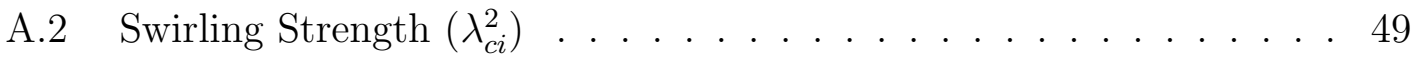

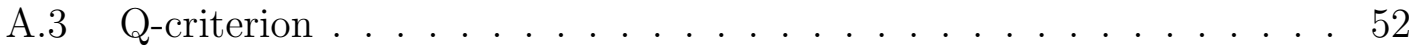

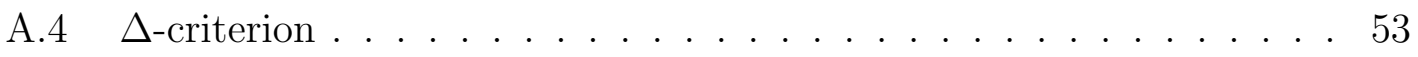

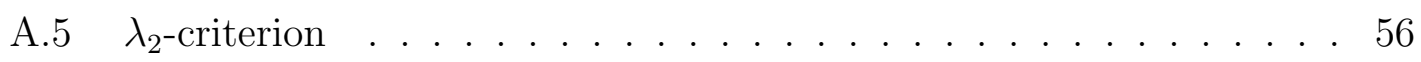

B Calculation Time for Each Criteria $\quad 58$

$\begin{array}{ll}\text { C Frame Selection } & 59\end{array}$

D Threshold Selection for Front and Back Frame of Turbine $\quad 61$ 


\section{List of Figures}

1.1 Turbulent energy cascade. . . . . . . . . . . . . . 2

1.2 Streamline trajectories based on the second and third invariant of the VGT (Perry and Chong [33]). . . . . . . . . . . . . 13

2.1 Wind tunnel inlet displaying a passive grid (group of diamond shape objects) and strakes (9 plexiglass elements vertically placed) 20

2.2 Wind turbine array side view. . . . . . . . . . . . . . 21

2.3 Wind turbine array top view. . . . . . . . . . . . . . . 21

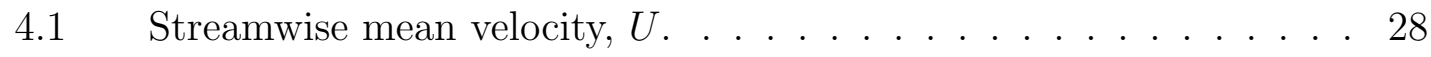

4.2 Mean kinetic energy flux, $-\overline{u^{\prime} v^{\prime}} U \ldots \ldots$. . . . . . . . . . . . 29

4.3 Instantaneous velocity field. . . . . . . . . . . . . . . . 30

4.4 Instantaneous turbulent kinetic energy, $\tilde{k}=\frac{1}{2}\left(u^{\prime 2}+v^{\prime 2}+w^{\prime 2}\right) . \quad \ldots 30$

$4.5 \quad$ Vorticity, $\omega \ldots \ldots \ldots \ldots \ldots \ldots$

4.6 Swirling strength, $\lambda_{c i}^{2} \ldots \ldots \ldots \ldots$. . . . . . . . . . . . . .

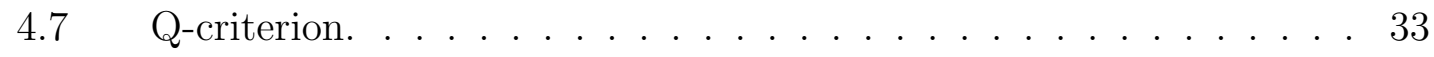

$4.8 \Delta$-criterion. . . . . . . . . . . . . . . 34

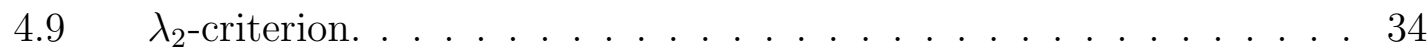

4.10 Galilean decomposition at 20\% overlaid with non-oriented swirling

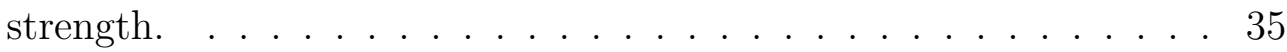


4.11 Galilean decomposition at $50 \%$ overlaid with non-oriented swirling strength. . . . . . . . . . . . . . . . . 36

4.12 Reynolds decomposition (showing $u^{\prime}$ ) overlaid with non-oriented swirling strength. . . . . . . . . . . . . . . . 37 


\section{Chapter 1}

\section{Introduction}

\subsection{Wind Energy}

Wind energy provides an increasingly significant portion of the global energy supply. By 2020, wind power is projected to account for $12 \%$ of global energy and by 2030 could supply as high as 20\% (Global Wind Energy Council 2012 [1]). Understanding the wake of the wind turbine array allows to understand the behavior of the velocity deficit and turbulence. The flow field provides insight on details of wind power generation as well as loading on the structure and power fluctuations. Vortical (also known as coherent) structures are central to turbulence. The location and strength of these structures directly relate to turbine structure loading and power fluctuations. The relation is clear when reviewing the turbulent energy cascade, as seen in Figure 1.1. In the turbulent energy cascade, the energy is plotted as a function of wave number, where low wave number represents high energy content. The large and high energy containing structures break into smaller and smaller structures where, at a small enough scale (known as the Kolmogorov dissipative scale), they are overcome by viscous effects, thus dissipating into internal energy. Energy, the dependent variable, equates to energy extraction potential as well as loading 
on the turbine structure. As larger and more numerous wind farms are built, the demand for an increase in efficiency drives studies such as the present investigation in search of maximizing power.

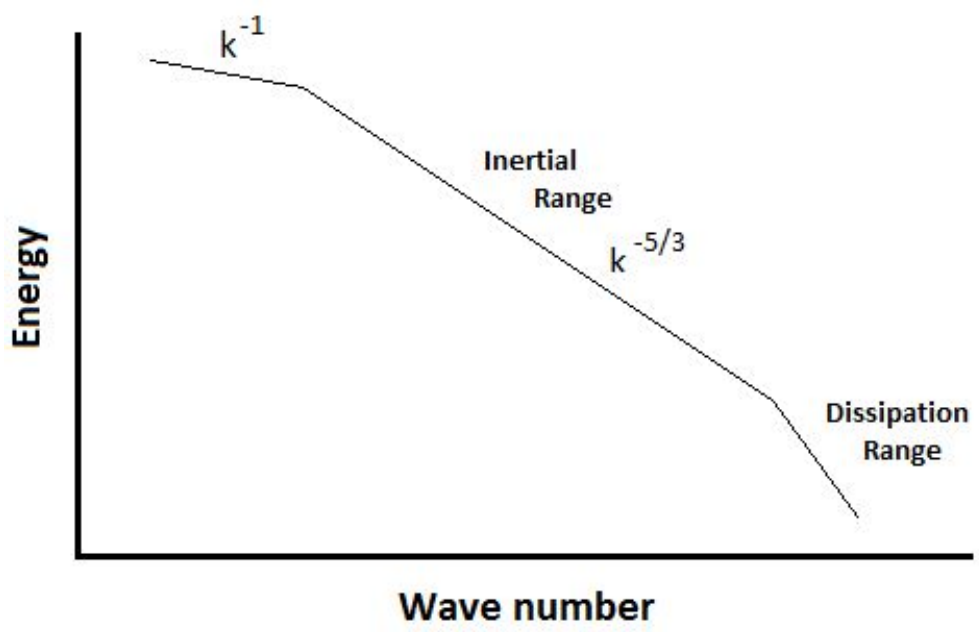

Figure 1.1: Turbulent energy cascade.

This study is focused on the identification of vortices located in the wake of a wind turbine array, specifically within the region of the infinite array. The utilization of the infinite array concept allows us to analyze the incoming and outgoing flow of the fourth row centerline turbine subject to periodic boundary conditions. The infinite array concept was reviewed by Chamorro and Porté-Agel [2], where boundary layer effects were studied in a wind tunnel using a 10 x 3 array of model wind turbines. It was found that below the top tip of the turbines (inside the turbine canopy), the turbulence statistics were shown to reach a point of equilibrium after the third row of turbines. The region inside the turbine canopy directly affects the turbine performance. Therefore, statistical quantities can be analyzed in relation to 
turbine performance, in the front and back of the fourth row centerline turbine in an array, simultaneously extending these results to the following rows in a wind farm.

Upon identification of vortex regions, analysis of the location and behavior of the vortices provides insight on designs mitigating loading on the turbine structure. Saranyasoontorn and Manuel [3] studied POD (proper orthogonal decomposition) modes and their relation to loading on the turbine structure. The key modes of loading on a wind turbine structure were found to be: flapwise (torsion of the blade) and edgewise bending moments at the blade root and fore-aft base bending moments of the tower. Edgewise blade bending loads are a result of the rotational speed of the turbine and the weight of the blade, while the flapwise and base bending moments are related to the loads resulting from the incoming flow. Fatigue accumulations have also been found to be related to levels of turbulence intensity (Rosen and Sheinman [4], Van Binh et al. [5]).

The near-wake region is a complex flow structure consisting of rotational effects of the blades (including tip vortices and root vortices), the flow from above the turbine (which assists with flow recovery), turbine array effects, and the flow around the mast. Zhang et al. [6], in a wind tunnel study of the wake of a single horizontalaxis wind turbine, found that tip vortices generated from the blades persist up to 3 rotor diameters downstream. Pedersen and Antoniou [7] completed a field study observing the wake of a wind turbine. Smoke emitting grenades were attached to the blades and on the mast at hub height. Helical tip vortices generated from the blades were observed. The tip vortices were found to persist longer with increasing 
rotational speed. Moving downstream the tip vortices became unstable and lost their circular shape. The continuity of the tip vortices was disrupted in the bottom tip region due to the tower. The smoke trace uncovered the root vortex resulting from the blade root (at hub height), where it was found to disperse quickly (after a half a revolution).

The transfer of energy into and within the wind turbine array is vital in understanding the flow behavior and optimization of power extraction in wind farms. Cal et al. [8] stated that, in large arrays, there is entrainment of kinetic energy from the flow above the wind turbines. Entrainment is part of a process to recover the wake, increasing power extraction potential in the wind turbine array (Hamilton et al. [9]). To further understand this concept, the Reynolds-Averaged Navier-Stokes boundary layer equation in subscript notation for high Reynolds number is given,

$$
U_{j} \frac{\partial U_{i}}{\partial x_{j}}=-\frac{1}{\rho} \frac{d P}{d x_{i}}-\frac{\partial}{\partial x_{j}} \overline{u_{i}^{\prime} u_{j}^{\prime}}-\overline{f_{x}}
$$

where $U$ is the velocity, $P$ is the pressure, $\rho$ is the fluid density, the overbar and capital letters represent averaging, and primes denote fluctuation. The unsteady term in the material derivative has been removed due to the assumption of a steady flow. The viscous effects have been assumed to be small due to flow measurements being a sufficient distance from the wall. The $\overline{f_{x}}$ forcing term represents the thrust effect of the wind turbine caused by the time-dependent pressure and viscous forces acting at the moving blade-air interface in the streamwise direction. The forcing term has been averaged over time to eliminate periodic time-dependence from rotation of the blades. Equation 1.1 is a result of neutrally buoyant flow in a wind 
tunnel, neglecting Coriolis force effects and buoyancy terms which would otherwise be important in field conditions.

Multiplying the momentum equation (Equation 1.1) by the mean velocity results in the mean kinetic energy equation,

$$
U_{j} \frac{\partial \frac{1}{2} U_{i}^{2}}{\partial x_{j}}=-\frac{1}{\rho} U_{i} \frac{d P}{d x_{i}}+\overline{u_{i}^{\prime} u_{j}^{\prime}} \frac{\partial U_{i}}{\partial x_{j}}-\frac{\partial \overline{u_{i}^{\prime} u_{j}^{\prime}} U_{i}}{\partial x_{j}}-\overline{f_{x} U_{i}}
$$

where $\overline{f_{x} U_{i}}$ represents the power density extracted from the flow by the wind turbine, $\overline{u_{i}^{\prime} u_{j}^{\prime}} \frac{\partial U_{i}}{\partial x_{j}}$ is the production of turbulent kinetic energy, and $\frac{\partial \overline{u_{i}^{\prime} u_{j}^{\prime}} U_{i}}{\partial x_{j}}$ is the flux of mean kinetic energy.

In a classical boundary layer, within the inner region (below $20 \%$ of the boundary layer thickness), the advection and pressure gradient terms are negligible. The atmospheric boundary layer thickness is about $1000 \mathrm{~m}$ and the wind turbines can stand at a height of $150 \mathrm{~m}$ (Cal et al. [8]). Therefore, it is appropriate to consider the turbine canopy within the inner region of the atmospheric boundary layer. The resulting mean kinetic energy equation inside a turbine canopy is

$$
\overline{u_{i}^{\prime} u_{j}^{\prime}} \frac{\partial U_{i}}{\partial x_{j}}-\frac{\partial \overline{u_{i}^{\prime} u_{j}^{\prime}} U_{i}}{\partial x_{j}}-\overline{f_{x} U_{i}}=0 .
$$

This leads to the conclusion that flow energy lost due to production of turbulent kinetic energy and the power density extraction is balanced with the flux of mean kinetic energy. Furthermore and using a conditional sampling technique, Hamilton et al. [9] stated that turbulent bursts in the positive streamwise direction moving 
downward are represented by sweeps. The presence of sweeps, which are indicated by the signs of $u^{\prime}>0$ and $v^{\prime}<0$, were shown to be greatest above the wind turbine array. This means that the dominant source of flux of mean kinetic energy entrains from above the turbine canopy to recover the lost flow momentum.

\subsection{Vortex Identification Methods}

In the field of vortex identification, there is no accepted definition for a vortex. In general, one can consider a vortex as "...the rotating motion of a multitude of material particles around a common center", according to Lugt [10]. The present study compares vortex identification techniques in the wake of a wind turbine array, namely the Q-criterion, $\Delta$-criterion, and $\lambda_{2}$-criterion. The resulting performance of each technique will improve the selection process of an appropriate technique in accordance with the flow dynamics observed within this study. Performance will be based on the ability to accurately identify vortex regions and the time required to complete calculation and output results. Further analysis in studying the vortex location, strength, and behavior is completed using Galilean and Reynolds decomposition, vorticity, and swirling strength. Knowledge of this information is necessary in designing for wind turbine structure loading and maximizing power extraction.

\subsubsection{Decomposition Techniques}

Galilean analysis uses the principle of decomposing the instantaneous velocity, $\tilde{u}$, based on a constant convection velocity, $U_{c}$, and fluctuating velocity, $u_{c}$, 


$$
\tilde{u}=U_{c}+u_{c}
$$

The premise of this method is based on vortices existing within the fluctuating velocity field. The appropriate convection velocity is determined by incrementally adjusting percentages (say by $5 \%$ from $0 \%$ to $100 \%$ ) of a maximum convection velocity. When subtracting the convection velocity percentages from the instantaneous velocity field, the fluctuating velocity field containing swirling motions travelling near the specified convection velocity is visualized in a vector plot.

Reynolds decomposition is a technique for decomposing the instanteous velocity, $\tilde{u}$, into the mean, $U$, and fluctuation, $u^{\prime}$,

$$
\tilde{u}=U+u^{\prime}
$$

Subtraction of the local mean velocity from the instantaneous velocity field results in the fluctuating velocity field, displaying all swirling motions convecting at the local mean velocity.

In Adrian et al. [11], vortices were identified in a fully developed pipe flow using Reynolds and Galilean decomposition. It was found that Reynolds decomposition was successful in revealing small-scale vortices, since most vortices were convecting close to the mean velocity. The Galilean decomposition required using a range of convection velocities, resulting in the identification vortex cores within the flow field. One advantage of the Galilean decomposition, as opposed to the Reynolds 
decomposition, was that relative shears between adjacent momentum structures in the flow were preserved.

\subsubsection{Velocity Gradient Tensor Techniques}

To understand the strengths of the $\mathrm{Q}, \Delta$, and swirling strength vortex identification techniques, the velocity gradient tensor (VGT) is central. The VGT describes the velocity at any point in a flow field. The VGT is obtained via a Taylor series expansion of the velocity field to the linear order,

$$
u_{i}=A_{i}+A_{i j} x_{j}
$$

where Perry and Chong [12] derived the VGT for an incompressible flow as $A_{i j}$. For an incompressible flow, $A_{i}$ equates to zero. The three-dimensional form of the VGT is

$$
\nabla u=\frac{\partial u_{i}}{\partial x_{j}}=\left(\begin{array}{ccc}
\frac{\partial u}{\partial x} & \frac{\partial v}{\partial x} & \frac{\partial w}{\partial x} \\
\frac{\partial u}{\partial y} & \frac{\partial v}{\partial y} & \frac{\partial w}{\partial y} \\
\frac{\partial u}{\partial z} & \frac{\partial v}{\partial z} & \frac{\partial w}{\partial z}
\end{array}\right) .
$$

The formulation of the VGT, shown in Equation 1.7, is used in derivation of the vortex identification techniques. The VGT is composed of a strain rate, $S_{i j}$, and rotation rate, $\Omega_{i j}$, tensor:

$$
\nabla u=S_{i j}+\Omega_{i j} .
$$


The strain rate and rotation rate tensor are given by,

$$
S_{i j}=\frac{1}{2}\left(\nabla u+(\nabla u)^{T}\right)=\frac{1}{2}\left(\frac{\partial u_{i}}{\partial x_{j}}+\frac{\partial u_{j}}{\partial x_{i}}\right)
$$

and

$$
\Omega_{i j}=\frac{1}{2}\left(\nabla u-(\nabla u)^{T}\right)=\frac{1}{2}\left(\frac{\partial u_{j}}{\partial x_{i}}-\frac{\partial u_{i}}{\partial x_{j}}\right) .
$$

The rotation rate tensor can be written using vorticity, $\omega_{k}$,

$$
\Omega_{i j}=\frac{1}{2} \omega_{k}
$$

A vorticity criterion $(|\omega|>0)$ has been used as a vortex identification technique by many including Metcalfe et al. [13], Hussain [14], and Bisset et al. [15]. The vorticity criterion was successful when studying free shear flows, but not guaranteed. The weakness of the vorticity criterion was present in cases where the shear was of comparable magnitude to the vorticity, similar conclusions were made in the following studies. In isotropic turbulence, tubular-like vortices ("worms") were identified using the vorticity criterion (Jiménez et al. [16], Dubief and Delcayre [17]). The structures appeared more like patches or sheets of vorticity rather than resulting in a clear tubular form. Studies of vortical structures in near wall flows resulted in high magnitudes of vorticity, but difficulties arose when attempting to differentiate between vortical structures and regions of high shear (Brooke and Hanratty [18], Robinson [19], Adrian et al. [11], Dubief and Delcayre [17]). Robinson [19] and Adrian et al. [11] utilized streamline and vector plots to supplement their vorticity results in order to identify swirling regions in near wall flows. In a mixing layer, Du- 
bief and Delcayre [17] found the vorticity criterion highlighting areas of high shear in the entrance region where no vortices are present, but downstream identified ribs and rolls where rotation was of a larger magnitude than shear. Comte et al. [20] were able to visualize roll-up and pairing of structures within the mixing layer of a solid-propellant rocket engine. In the backward facing step, regions of high shear were misrepresented as vortex regions (Dubief and Delcayre [17]). Haller [21] found similar behavior in a parallel shear flow containing high magnitudes of vorticity where no vortices were present.

The characteristic equation for the VGT leads to the Q-criterion. Arriving at the characteristic equation requires an eigen-decomposition of the VGT. The resulting characteristic equation for the VGT is

$$
\lambda^{3}-\frac{\partial u_{i}}{\partial x_{i}} \lambda^{2}+\left(\frac{\partial u_{i}}{\partial x_{i}} \frac{\partial u_{j}}{\partial x_{j}}-\frac{\partial u_{i}}{\partial x_{j}} \frac{\partial u_{j}}{\partial x_{i}}\right) \frac{\lambda}{2}-\operatorname{det}\left(\frac{\partial u_{i}}{\partial x_{j}}\right)=0
$$

also written as

$$
\lambda^{3}-P \lambda^{2}+Q \frac{\lambda}{2}-R=0
$$

where $\lambda$ corresponds to the eigenvalues, the first invariant $P=-\frac{\partial u_{i}}{\partial x_{i}}$, the second invariant $Q=\frac{\partial u_{i}}{\partial x_{i}} \frac{\partial u_{j}}{\partial x_{j}}-\frac{\partial u_{i}}{\partial x_{j}} \frac{\partial u_{j}}{\partial x_{i}}$, and the third invariant $R=-\operatorname{det}\left(\frac{\partial u_{i}}{\partial x_{j}}\right)$ (where "det" represents the determinant). For an incompressible flow, Equation 1.12 reduces to:

$$
\lambda^{3}-\frac{\partial u_{i}}{\partial x_{j}} \frac{\partial u_{j}}{\partial x_{i}} \frac{\lambda}{2}-\operatorname{det}\left(\frac{\partial u_{i}}{\partial x_{j}}\right)=0
$$

also written as 


$$
\lambda^{3}-Q \frac{\lambda}{2}-R=0
$$

The second invariant less than zero, also known as the Q-criterion, represents the dominance of rotation over strain. The Q-criterion for an incompressible flow is

$$
Q=\frac{\partial u_{i}}{\partial x_{j}} \frac{\partial u_{j}}{\partial x_{i}}=\frac{1}{2}\left(\Omega_{i j} \Omega_{i j}-S_{i j} S_{i j}\right)>0
$$

Hunt et al. [22] introduced this criterion by applying two criteria to define eddy zones: (1) the second invariant of the VGT was to be less than a negative threshold value and (2) have a pressure minimum. The Q-criterion is also known as the elliptic version of the Okubo-Weiss criterion (Okubo [23], Weiss [24]). Relating the incompressible form of the Q-criterion (Equation 1.14) to pressure can be completed by taking the divergence of the Navier-Stokes equation and applying continuity,

$$
Q=\frac{1}{2}\left(\frac{1}{2} \omega^{2}-S_{i j} S_{i j}\right)=\frac{1}{2 \rho} \nabla^{2} p
$$

It can be seen, using the maximum principle of a harmonic function (Laplacian of the pressure), that the pressure maximum occurs at the boundary when $Q$ is positive and the pressure minimum occurs on the boundary if $Q \leq 0$, as pointed out by Dubief and Delcayre [17].

Jeong and Hussain [25] as well as Cucitore et al. [26] reviewed the Q-criterion in analytical flow fields: the Bödewadt vortex and the conically symmetric vortex. Cucitore et al. [26] also reviewed the unsteady inviscid radially stretched vortex. The Bödewadt vortex contains vortical motion down to the wall. The Q-criterion 
resulted in a negative value close to the wall, misrepresenting vortical motion. The conically symmetric vortex contains vortical motion while the Q-criterion resulted in negative values for the entire flow field. In the unsteady inviscid radially stretched vortex, the Q-criterion resulted in negative values at the core, misrepresenting the vortex core. The Burger's vortex tube model, another analytical flow field, was studied by Horiuti [27] and Jeong and Hussain [25] where, using the Q-criterion, the center of the vortex incorrectly resulted in negative values. From the results of the analytical flow fields, arguments can be made that the Q-criterion has weakness in identifying vortical motion when the strain is comparable to vorticity or in flows containing strong vortex core dynamics (Jeong and Hussain [25]). Dubief and Delcayre [17], in defense of the Q-criterion, addressed the inadequacies of the Qcriterion in the analytical flow fields, but argued that these are unlikely in common turbulent flows.

The Q-criterion was applied to isotropic turbulence, where clear vortex cores were visualized only after adjustment of non-zero threshold values (Horiuti [27], Chakraborty et al. [28], Dubief and Delcayre [17]). Dubief and Delcayre [17] found the Q-criterion successful in capturing detailed vortical structures in a mixing layer, channel flow, and a backward facing step. Using the Q-criterion in detached-eddy simulations for a M219 experimental cavity, Lawson and Barakos [29] were able to reveal turbulent content resulting from the door, leading edges, and door hinges.

By taking the discriminant of the characteristic equation (Equation 1.12) for the VGT, Vollmers [30] and Dallman [31] introduced the $\Delta$-criterion. For an incom- 
pressible flow, the criterion is

$$
\Delta=\frac{\left(\frac{\partial u_{i}}{\partial x_{j}} \frac{\partial u_{j}}{\partial x_{i}}\right)^{3}}{27}+\frac{\operatorname{det}\left(\frac{\partial u_{i}}{\partial x_{j}}\right)^{2}}{4}=\frac{Q^{3}}{27}+\frac{R^{2}}{4}>0
$$

where $\mathrm{Q}$ and $\mathrm{R}$ are the second and third invariant of the VGT, respectively. For $\Delta>0$, there is one real and two complex eigenvalues for the VGT (Spiegel and Liu [32]). This implies that the pattern of the streamlines are either closed or spiralled, as stated by Perry and Chong [33]. Using critical point analysis based on the Taylor expansion of the instantaneous velocity field (refer to Equation 1.6), Perry and Chong [33] described the streamline trajectory using the P-Q plot, where they defined $\mathrm{P}$ as the second invariant and $\mathrm{Q}$ as the third invariant of the VGT, as shown in Figure 1.2.

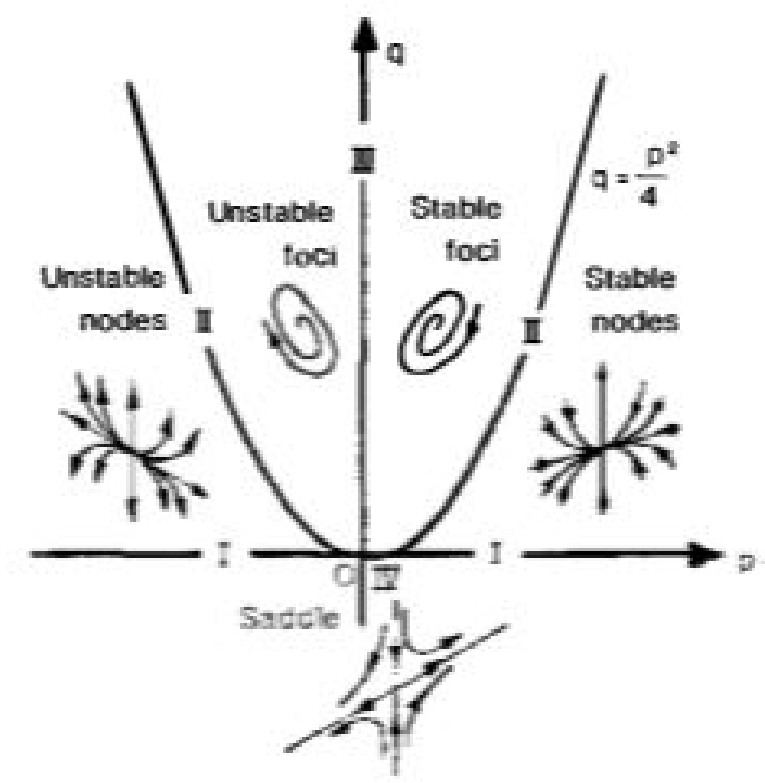

Figure 1.2: Streamline trajectories based on the second and third invariant of the VGT (Perry and Chong [33]).

In the case of $\Delta>0$, this corresponds to "foci" critical points representing closed 
or spiralled streamline trajectories.

Dallman et al. [34] showed that vortex cores can be located using the $\Delta$-criterion in laminar wakes behind a sphere in subsonic flow, the transitional transonic flow around a round-edged delta wing and the laminar hypersonic flow past a double ellipsoid. Jeong and Hussain [25] reviewed the $\Delta$-criterion in a circular jet at the time of roll-up. It was shown that the $\Delta$-criterion displayed obscured details of the vortex structure and over-estimated its size. In isotropic turbulence, the $\Delta$-criterion identified vortical structures, but did not result in clear vortex tubes (Chakraborty et al. [28], Jeong and Hussain [25]).

Further analysis in the complex eigenvalues of the VGT resulted in the swirling strength criterion. The canonical (eigenvalue) matrix for complex eigenvalues is written as $A^{\prime}$ (Zhou et al. [35], Chong et al. [36]):

$$
A^{\prime}=\left(\begin{array}{ccc}
\lambda_{r} & 0 & 0 \\
0 & \lambda_{c r} & \lambda_{c i} \\
0 & -\lambda_{c i} & \lambda_{c r}
\end{array}\right)
$$

The swirling strength criterion $\left(\lambda_{c i}^{2}>0\right.$ ), introduced by (Zhou et al. [35]), was defined as the square of the imaginary portion of the complex conjugate eigenvalue $\left(\lambda_{c r} \pm \lambda_{c i}\right)$ of the VGT. Eigenvalues resulting from the eigen-decomposition of the 3D VGT are either three real $\left(\lambda_{r}\right)$, or one real and a complex conjugate containing a complex real $\left(\lambda_{c r}\right)$ and complex imaginary value $\left(\lambda_{c i}\right)$ (Spiegel and Liu [32]). Zhou et al. [35] expressed the streamline trajectories based on the coordinate system $y_{1}, y_{2}, y_{3}$ 
defined by the eigenvectors of the VGT, $v_{r}, v_{c r}, v_{c i}$ :

$$
\begin{aligned}
& y_{1}(t)=C_{r} \exp \lambda_{r} t \\
& y_{2}(t)=\exp \lambda_{c r} t\left[C_{c}^{(1)} \cos \left(\lambda_{c i} t\right)+C_{c}^{(2)} \sin \left(\lambda_{c i} t\right)\right] \\
& y_{2}(t)=\exp \lambda_{c r} t\left[C_{c}^{(2)} \cos \left(\lambda_{c i} t\right)-C_{c}^{(1)} \sin \left(\lambda_{c i} t\right)\right]
\end{aligned}
$$

where $C_{r}, C_{c}^{(1)}$, and $C_{c}^{(2)}$ are constants. From Equation 1.18 we can see that $\lambda_{r}$ represents the stretching of the vortex, $\lambda_{c r}$ is the size of the swirling motion, and $2 \pi / \lambda_{c i}$ is the time required to complete one revolution. The swirling strength criterion does not contain directional information for the rotation of the vortex. A swirling strength $\left(\lambda_{c i}^{2}\right)$ and vorticity $(\omega)$ comparison is necessary to obtain directional information (utilized in this study).

Zhou et al. [35] introduced the swirling strength criterion when studying the evolution of a single hairpin vortex-like structure. The structure was studied using DNS in the mean turbulent flow field of a channel at a low Reynolds number. Non-zero threshold values were utilized, increasing clarity of the hairpin structure. Adrian et al. [11] showed that the swirling strength criterion was successful in capturing small-scale vortices in high Reynolds number pipe flow. Chakraborty et al. [28] discussed the weakness of how the swirling strength criterion with a zero threshold had difficulty identifying and representing the vortex core for both a consolidated jet and isotropic turbulence. 


\subsubsection{Critical Points via Hessian of the Pressure}

The Hessian of the pressure, $H_{p}$, is the gradient of the gradient of pressure, $p$, written as (Jeong and Hussain [25]):

$$
H_{p}=\nabla(\nabla p)=\frac{\partial p}{\partial x_{i} \partial x_{j}}
$$

A critical point, representing a pressure minimum, requires two eigenvalues of the Hessian of the pressure to be positive. Based on this concept, the $\lambda_{2}$-criterion $\left(\lambda_{2}<0\right)$ was introduced by Jeong and Hussain [25] for incompressible flows. The derivation of the criterion will be based on notation found in Jeong and Hussain [25].

Obtaining a relationship to the Hessian of the pressure requires taking the gradient of the Navier-Stokes equation:

$$
a_{i, j}=-\frac{1}{\rho} p_{, i j}+\nu u_{i, j k k}
$$

where the Hessian of pressure is $p_{, i j}$. The resulting acceleration gradient, written as $a_{i, j}$, is composed of a symmetric part (with $S_{i j}$ contained in the material derivative) and an anti-symmetric part (with $\Omega_{i j}$ contained in the material derivative):

$$
a_{i, j}=\left[\frac{D S_{i j}}{D t}+\Omega_{i k} \Omega_{k j}+S_{i k} S_{k j}\right]+\left[\frac{D \Omega_{i j}}{D t}+\Omega_{i k} \Omega_{k j}+S_{i k} S_{k j}\right]
$$

where the anti-symmetric part results in the vorticity transport equation. The antisymmetric part is ignored because it does not contribute in detecting a pressure minimum since the pressure term is zero. The symmetric portion is 


$$
\frac{D S_{i j}}{D t}-\nu S_{i j, k k}+\Omega_{i k} \Omega_{k j}+S_{i k} S_{k j}=-\frac{1}{\rho} p_{, i j}
$$

where $\frac{D S_{i j}}{D t}$ represents unsteady irrotational strain, $\nu S_{i j, k k}$ contains the viscous effects, $\Omega_{i k} \Omega_{k j}$ is the rotation rate tensor product, $S_{i k} S_{k j}$ is the strain rate tensor product, and $\frac{1}{\rho} p_{, i j}$ is the pressure term containing the Hessian of the pressure.

To obtain a local pressure minimum indicator via critical points of the Hessian of the pressure, Jeong and Hussain [25] ignored terms from Equation 1.22 that would adversely affect a local pressure minimum associated with swirling motion. In Stoke's flow, vortices can occur, but no local pressure minimum is resulted, and thus leading to a neglecting of the viscous effects, $\nu S_{i j, k k}$. The unsteady irrotational strain $\frac{D S_{i j}}{D t}$ is also ignored due to the possibility of producing a pressure minimum through flow expansion without rotation or otherwise swirling motion. The resulting equation leads to a local pressure minimum indicator defined by Jeong and Hussain [25] as $A=S^{2}+\Omega^{2}$. $\lambda_{2}$ is the intermediate eigenvalue of $A\left(\lambda_{1} \geq \lambda_{2} \geq \lambda_{3}\right)$. $\lambda_{2}<0$ corresponds to the identification of a vortex region.

The $\lambda_{2}$-criterion was found to be successful in identifying vortex cores in analytical flow fields such as the tornado model (Jeong and Hussain [25]), Bödewadt vortex (Cucitore et al. [26]), and unsteady inviscid radially stretched vortex (Cucitore et al. [26]). Jeong and Hussain [25] found that $\lambda_{2}$ resulted in a well-defined vortical ring structure in a circular jet at roll-up. The $\lambda_{2}$-criterion was able to show the ribs and rolls in the mixing layer (Jeong and Hussain [25], Dubief and Delcayre [17]). In isotropic turbulence, the adjustment of non-zero threshold values 
was necessary to identify clear tubular structures (Horiuti [27], Dubief and Delcayre [17], Chakraborty et al. [28]). Structures were also identified in channel flow and the backward facing step using the $\lambda_{2}$-criterion, but the structures contained noise (Dubief and Delcayre [17]).

Jeong and Hussain [25] stressed the need for Galilean invariance which lead to the $\lambda_{2}$-criterion, while Haller [21] stated that Galilean invariance is insufficient. Haller [21] explained that in rotating flows, where the speed of rotation is high, frame dependent criteria will not properly identify the vortex, but rather consider the whole space as a single vortex. Galilean or Eulerian techniques are known to have weaknesses, but are found to be successful in many turbulent flows, as stated in the applications of the criteria.

The aforementioned techniques are applied to SPIV data in the wake of a wind turbine array to identify vortex regions. Accurately identified vortices will be comparable to those found in field studies (Pedersen and Antoniou [7]) and wind tunnel experiments (Zhang et al. [6]). The successful techniques will provide future studies the tools necessary to efficiently and effectively interpret the dynamics observed in a wind energy application. 


\section{Chapter 2}

\section{Experimental Setup}

The Portland State University closed circuit wind tunnel has a 9:1 contraction ratio, and a test section 5 meters long and $0.8 \mathrm{~m} \mathrm{x} 1.2 \mathrm{~m}$ in cross-section. The wind tunnel has a steel framework with fixed Schlieren-grade annealed float glass surfaces to allow for non-intrusive laser-based velocity field measurements. As seen in Figure 2.1, a passive grid is utilized in generating a comparable turbulence intensity to field studies (12\% to 15\%). Strakes are located forward of the passive grid to shape the flow in generating an atmospheric-like boundary layer. The strakes are made of $0.0125 \mathrm{~m}$ thick plexiglass. Nine strakes are placed vertically at a spacing of $0.136 \mathrm{~m}$ and positioned $0.5 \mathrm{~m}$ downstream. Surface roughness is added in the form of small-diameter chains to extend the influence of the high shear zone, further shaping the boundary layer. The chains have a diameter of $0.0075 \mathrm{~m}$ and are spaced $10.8 \mathrm{~cm}$ streamwise. The boundary layer thickness $\delta$ is approximately $0.35 \mathrm{~m}$ with

a free stream velocity of $6 \mathrm{~m} / \mathrm{s}$ leading to a Reynolds number based on $\delta$ of $1.9 \times 10^{5}$. 


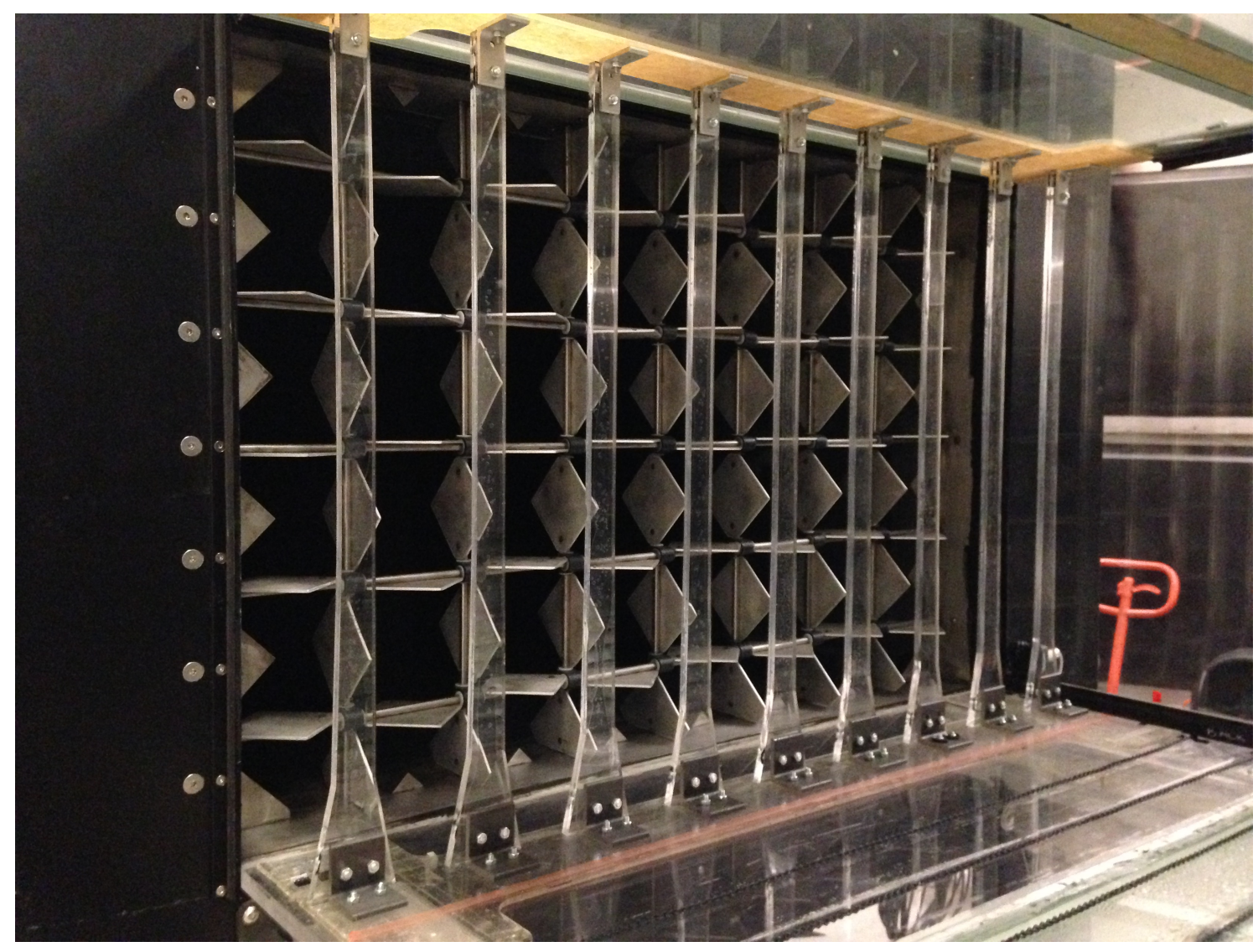

Figure 2.1: Wind tunnel inlet displaying a passive grid (group of diamond shape objects) and strakes (9 plexiglass elements vertically placed)

Model turbines are positioned in an array form, 4 streamwise and 3 spanwise (as seen in Figure 2.2 and 2.3). The scaled turbine models were manufactured in-house. Based on full scale turbines with a 100m rotor diameter and a 100m hub height, the scaled models are at 1:830 scale. The rotor blades are steel sheets laser cut to shape and are $0.0005 \mathrm{~m}$ thick. The blades are shaped using a die press. The die press was designed in-house to produce a 15 degree pitch from the plane of the rotor and a 10 degree twist at the tip. 


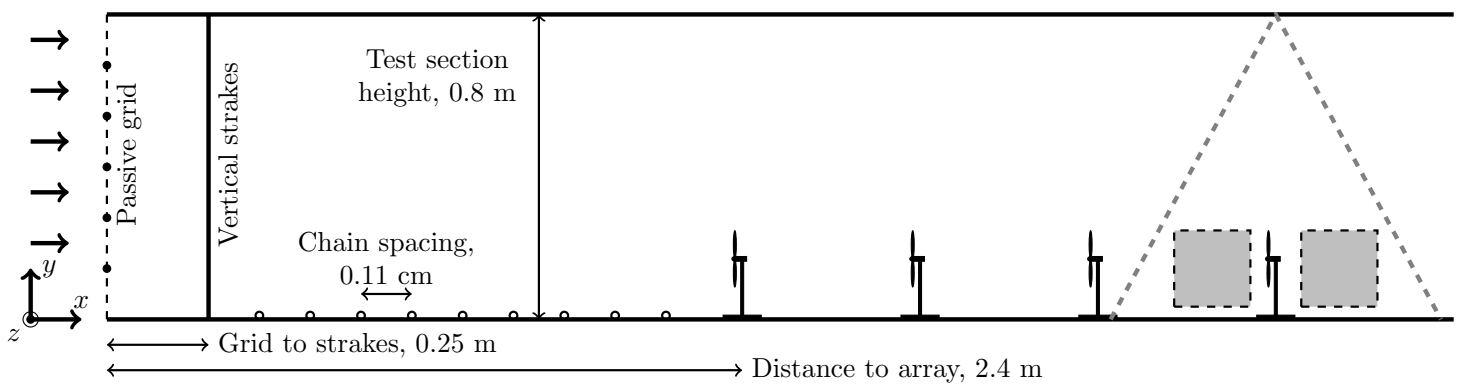

Figure 2.2: Wind turbine array side view.

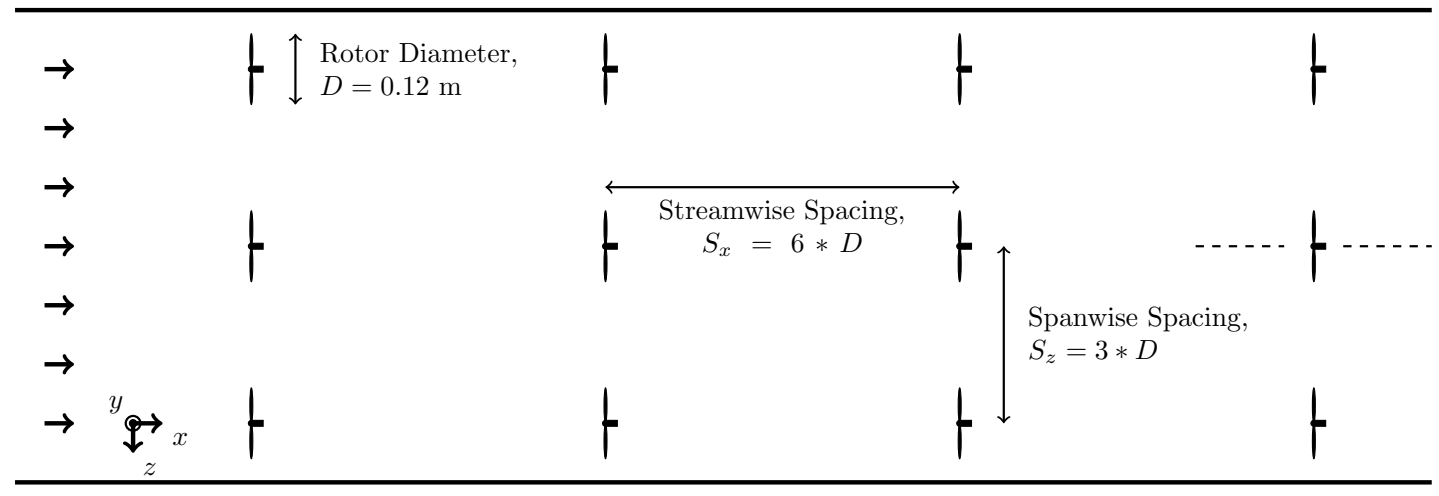

Figure 2.3: Wind turbine array top view.

The flow field was measured using stereographic particle image velocimetry (SPIV). Data was collected using two windows, directly upstream and downstream of the centerline exit row turbine in the array. The SPIV system model was LaVision and the whole system consisted of an Nd:Yag (532 nm, 1200mJ, 4ns duration) double-pulsed laser and four 4MP ImagerProX CCD cameras positioned in pairs directed towards the windows described above. Neutrally buoyant fluid particles of diethylhexyl sebecate were added to the flow and allowed to mix thoroughly. The addition of the fluid particles was constant to allow for consistent data resolution. The laser sheet was positioned at less than 5mrad divergence angle and was about $0.001 \mathrm{~m}$ thick. The measurement windows were $0.23 \mathrm{~m} \times 0.23 \mathrm{~m}$ with a $1.5 \mathrm{~mm}$ vector 
resolution. The measurement uncertainty was within 3 percent where the greatest error would pertain to the span-wise component, which was only used for the instantaneous turbulent kinetic energy calculation. 2000 SPIV image sets were collected at each measurement location. A multi-pass FFT based correlation algorithm was utilized in processing the raw data into vector fields. This was accomplished by reducing the size of the interrogation windows with a 50 percent overlap, twice at $64 \times 64$ and once at $32 \times 32$ pixels. The delay between image pairs was $130 \mathrm{mi}-$ croseconds leading to an average particle displacement of 8 pixels. Erroneous vectors generated through the processing was on the order of 1 percent of the total vectors processed. These vectors were found when analyzing the data by visual detection of non-physical peak regions in contour plots. These vectors were replaced using Gaussian interpolation of accurate neighboring vectors. For more information on the experiment conditions and data processing, see Hamilton et al. [37]. 


\section{Chapter 3}

\section{Analysis Methodology}

\subsection{Two-Dimensional Truncation}

The SPIV data contains streamwise $(u)$, vertical $(v)$, and spanwise $(w)$ velocities. The position data is two-dimensional, resulting in the streamwise $(x)$ and vertical (y) components. The quantities contained in the data lead to a truncation of the VGT that can be used in the analysis of the flow field. The resulting VGT for this study is

$$
\nabla u=\frac{\partial u_{j}}{\partial x_{i}}=\left(\begin{array}{cc}
\frac{\partial u}{\partial x} & \frac{\partial v}{\partial x} \\
\frac{\partial u}{\partial y} & \frac{\partial v}{\partial y}
\end{array}\right) .
$$

The resulting canonical (eigenvalue) matrix contains either two real eigenvalues,

$$
\left(\begin{array}{cc}
\lambda_{r} & 0 \\
0 & \lambda_{r}
\end{array}\right),
$$

or the complex conjugate eigenvalues (Adrian et al. [11]),

$$
\left(\begin{array}{cc}
\lambda_{c r} \pm \lambda_{c i} & 0 \\
0 & \lambda_{c r} \pm \lambda_{c i}
\end{array}\right)
$$


The data not gathered, pertaining to gradients in the spanwise direction, contain the stretching of the vortex structure associated with the third real eigenvalue in the complex eigenvalue matrix (Equation 1.19). This means that the swirling motion of the vortices in the $x-y$ plane generated by the incoming flow and wind turbine interaction can be identified, while the full helical form of the tip vortices cannot be resulted. Therefore, the physics describing the swirling motion are retained within the two-dimensional VGT.

Adrian et al. [11], gathered two-dimensional SPIV data in high Reynolds number pipe flow $\left(R e_{D}=50,000\right)$. They identified vortical structures using a twodimensional swirling strength algorithm. The pipe flow study is comparable to the present study due to the high Reynolds number and high shear magnitudes.

Jeong and Hussain [25] compared Q-criterion, $\Delta$-criterion, and $\lambda_{2}$-criterion for planar flows. It was found that there were similarities between the criterion for two-dimensional cases of the VGT. Consider a VGT for a planar flow,

$$
\nabla u=\left(\begin{array}{cc}
a & b \\
c & -a
\end{array}\right) .
$$

The resulting characteristic equation is $\lambda^{2}+Q=0$, where $\lambda$ represents the eigenvalues and $Q=-a^{2}-b c$. This leads us to the conclusion that when $Q$ is positive, the eigenvalues are complex (equivalent to the $\Delta$-criterion) since the resulting eigenvalues are $\lambda= \pm(-Q)^{\frac{1}{2}}$. The $A$ matrix used in the $\lambda_{2}$-criterion for planar flows is 


$$
S^{2}+\Omega^{2}=\left(\begin{array}{cc}
a^{2}+b c & 0 \\
0 & a^{2}+b c
\end{array}\right) .
$$

The resulting eigenvalues for the two-dimensional formulation indicate that when $\lambda_{2}$ is negative, it is equivalent to saying $Q>0$, where a negative $\lambda_{2}$ requires $a^{2}+b c<0$.

To convert the $\lambda_{2}$-criterion to a two-dimensional algorithm, we must determine the local minimum for the resulting two-dimensional Hessian of the pressure. For a local minimum to exist in a two-dimensional Hessian, the Hessian must be positive definite (Strang [38]). A positive definite Hessian means the eigenvalues are all positive. In this case, the Hessian of the pressure is negated in the formulation (see Equation 1.22). This requires that Equation 3.5 must have two negative eigenvalues. Equation 3.5 results in two real and equal eigenvalues, where obtaining a positive definite Hessian of the pressure (local minimum) requires $a^{2}+b c<0$, in agreement with the analysis by Jeong and Hussain [25].

\subsection{Method of Calculation}

The two-dimensional algorithms were developed using Matlab R2012a. The Matlab code for each criteria can be found in Appendix A. The gradients were calculated using a first order central difference technique. An un-optimized code was utilized and run on a laptop. Computational time for each vortex identification technique will be used in the performance comparison (see Appendix B for the calculation times). The criteria utilized in this study will be subject to a zero value threshold. 


\subsection{Frame Selection}

The vortex identification techniques compared in this study are Galilean/Eulerian techniques. Galilean/Eulerian techniques are frame dependent, requiring a frame selection for analysis. One frame was selected for comparison of the vortex identification techniques. The frame selection process required review of 500 out of the 2000 frames of data utilizing the swirling strength algorithm (Appendix C contains 5 of these frames). Resulting vortex regions were compared to field studies. In field studies (Pedersen and Antoniou [7]), helical tip vortices are generated. Due to the turbulent nature of the flow, the periodic nature of the tip vortices, and speed of capturing images, some frames did not display strong helical tip vortices at the top and bottom tip region. The frame selected (frame 118) for comparison contained the helical tip vortices at the top tip and bottom tip. 


\section{Chapter 4}

\section{Results and Discussion}

\subsection{Results}

In the midst of a complex turbulent flow field, vortices convecting within the wind turbine array are observed and analyzed. Mean statistics, based on the ensemble average of the 2000 PIV frames, are initially presented to gather a general sense of the flow field, including the streamwise mean velocity $U$ and mean kinetic en-

ergy flux $-\overline{u^{\prime} v^{\prime}} U$. The instantaneous turbulent kinetic energy $\frac{1}{2}\left(u^{\prime 2}+v^{\prime 2}+w^{\prime 2}\right)$ and instantaneous velocity vector field are also included to describe the selected frame flow field and provide a comparison to the vortex identification techniques. The layout of plots will illustrate the incoming flow as shown in the first interrogation window in Figure 4.1 and thereafter its interaction with the last row center turbine, for which then the near wake is observed. Utilizing the concept of the infinite array, the incoming flow will be simultaneously referred to as the far wake of the last row center turbine. The axes are non-dimensionalized using the turbine rotor diameter. Following these results and their discussion, the vortex identification techniques will be applied to the flow and then compared. 
Figure 4.1 shows contours of the streamwise mean velocity immediately upstream and downstream of the last row center turbine. Within the turbine canopy $(y / D<1.5)$, the streamwise mean velocity is reduced due to the presence of the wind turbines. The incoming flow is observed to decrease in velocity at $x / D=-0.5$ (forward of the turbine rotor) and spread around the turbine. At hub height $(y / D=1)$ the velocity deficit is noticeable for the region in front of the turbine as well as in the near wake of the exit row turbine where the deficit is maximum. Wake recovery is shown to be increasing with downstream distance. The recovery is noticeably faster in the mast region $(y / D<0.5)$ as opposed to the hub region. At $y / D<0.4$, forward of the turbine, the velocity is shown to decrease moving closer to the wall due to the boundary layer effect in the far wake $(x / D>4$ or $>-2)$. In the near wake $(x / D<2)$, the flow is seen to experience inhibition of viscous wall effects in the mast region, a result of no flow attachment to the wall.
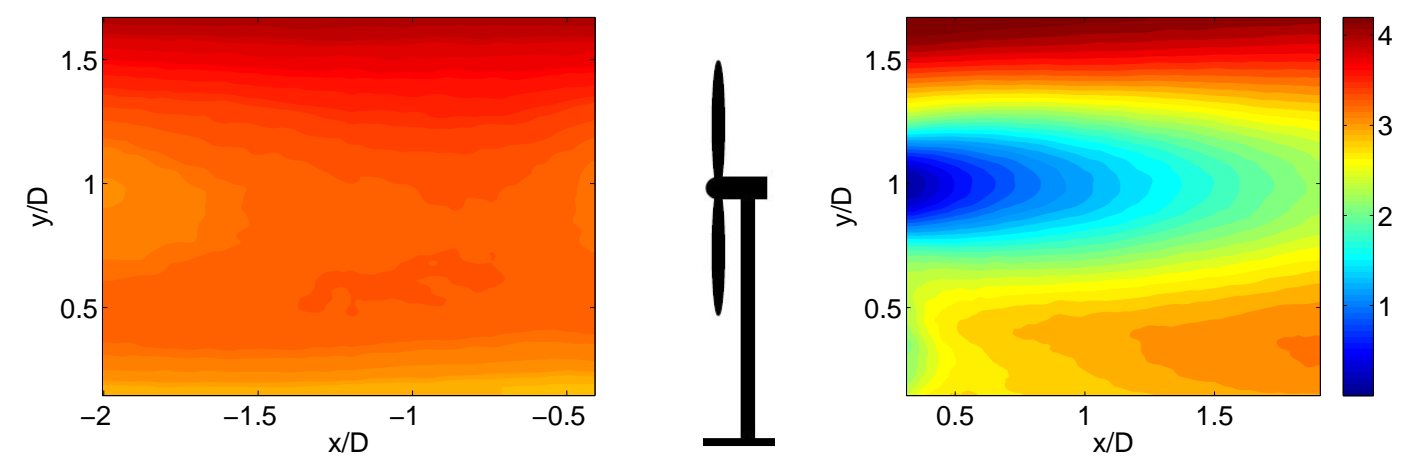

Figure 4.1: Streamwise mean velocity, $U$.

The mean kinetic energy flux (see Equation 1.3) of Figure 4.2 is related to the flow entrainment (Hamilton et al. [37]). Flow entrainment of mean kinetic energy is needed to replenish the flow momentum loss, as was discussed by Cal et al. [8] 
for a wind turbine array. The maximum gradient occurs immediately past 1D of the turbine. Entrainment occurs above the top and below the bottom tip region. The top tip region results in a higher magnitude of entrainment than the bottom tip region due to the higher flow velocity above the canopy. The flux is not as significant less than 1D downstream of the turbine due to the strong flow dynamics resulting from the blade motion. In comparing the front and back interrogation areas, a larger difference between the top and bottom tip exists aft of the turbine thus highlighting a greater flow energy deficit in the back of turbine region. Entrainment continues forward of the turbine indicating continued flow recovery extending to the following turbine.
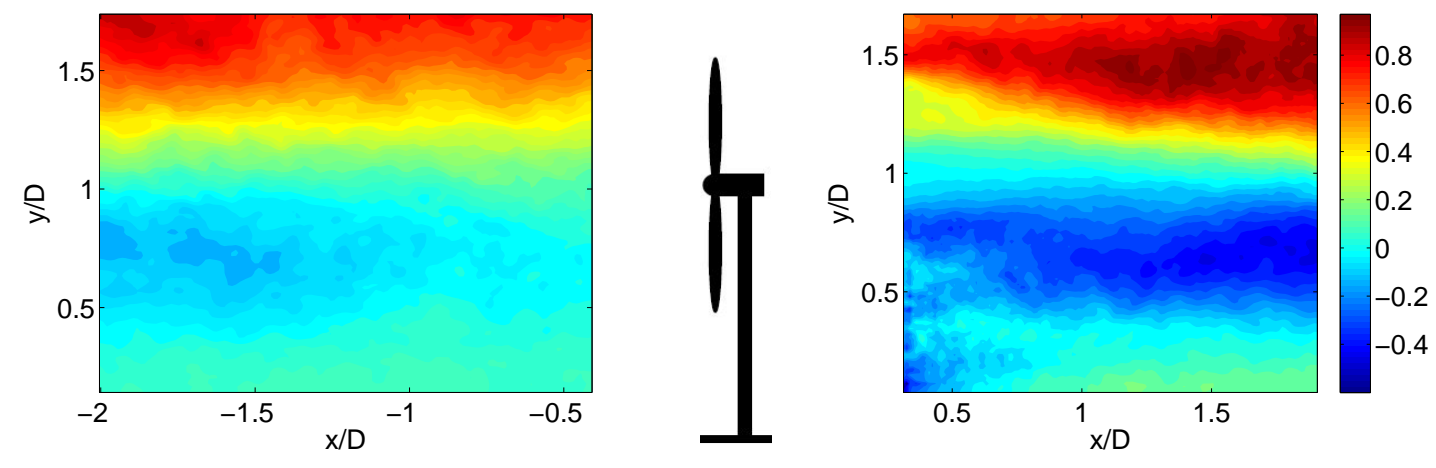

Figure 4.2: Mean kinetic energy flux, $-\overline{u^{\prime} v^{\prime}} U$.

Figure 4.3 is a vector map of the instantaneous velocity field. Herein, the selected frame is used for the different techniques (see Section 3.3 for more details on frame selection). The flow upstream of the turbine has a consistent flow direction with minor upward and downward vectors throughout the flow field. In the wake, the flow diverges from the center region directly behind the hub after contacting the turbine and meanders downstream. Directly behind the hub, there is a velocity 
deficit including velocity minima and backflow. Shearing behavior is noticeable from the bottom tip $(y / D=0.5)$, but in a lesser magnitude than the top tip due to the presence of the tower and lower incoming flow velocity in this region, as found in Figure 4.1.
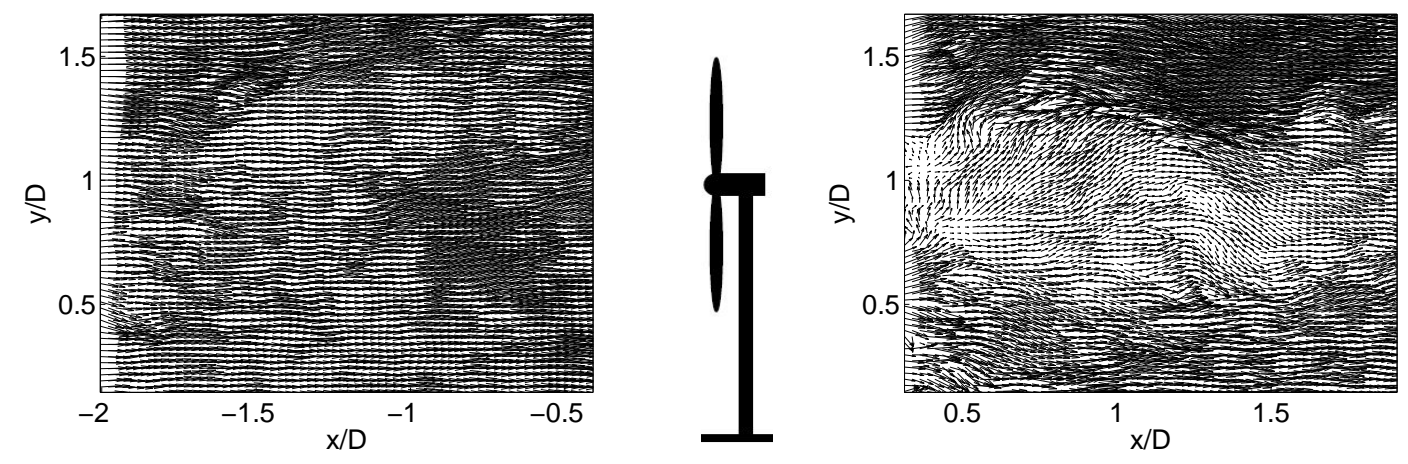

Figure 4.3: Instantaneous velocity field.

The instantaneous turbulent kinetic energy $\tilde{k}=\frac{1}{2}\left(u^{\prime 2}+v^{\prime 2}+w^{\prime 2}\right)$ is shown in Figure 4.4. The colorbar ranges of $\tilde{k}$ are adjusted in the front and back of the turbine to elucidate different features of the flow.
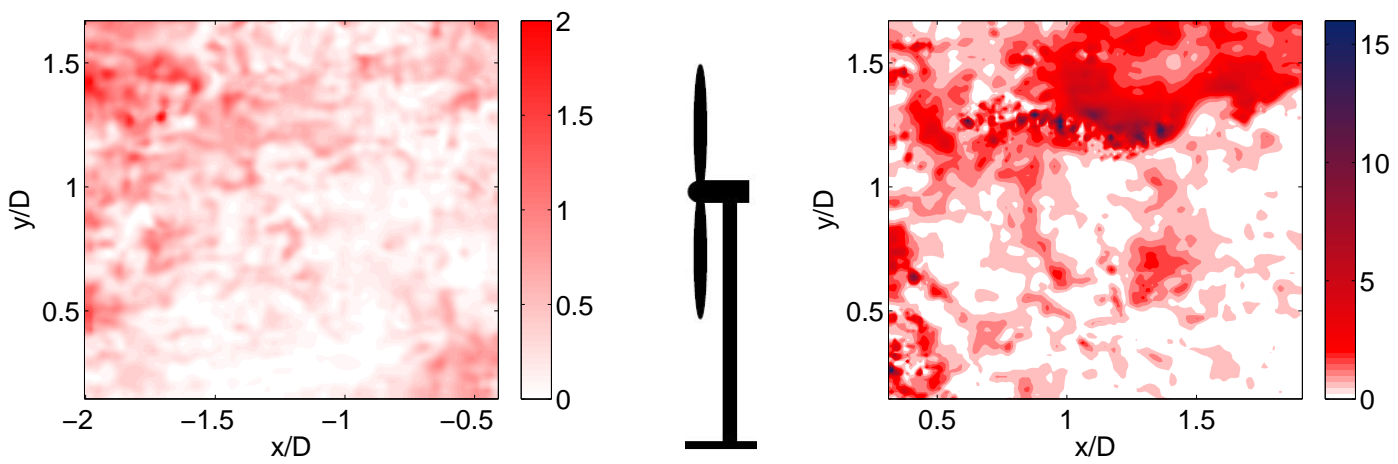

Figure 4.4: Instantaneous turbulent kinetic energy, $\tilde{k}=\frac{1}{2}\left(u^{\prime 2}+v^{\prime 2}+w^{\prime 2}\right)$. 
The peak magnitude of $\tilde{k}$ in front of the turbine is eight times smaller than that of the wake of the turbine. In the plane immediately in front of the turbine, low magnitudes are distributed over the plane, whereas after the turbine, high magnitudes are well aligned with the top tip of the rotor. This is due to the passage of the rotor which interacts with the flow and generates helical tip vortices. The helical tip vortices concentrate in the outer region of the rotor wake where they are sheared by the entraining flow.

Figure 4.5 is a plot of vorticity in the plane. The colorbar in Figure 4.5 includes 4 colors: 2 colors (black and blue) correspond to peak negative and positive magnitudes in the back of the turbine and the other 2 colors (orange and red) correspond to peak values in the front of the turbine. The same color scheme will be used for the positive and negative values in the vortex identification techniques. Regions of high vorticity are concentrated primarily in the path from the top tip and bottom tip in the wake of the turbine.
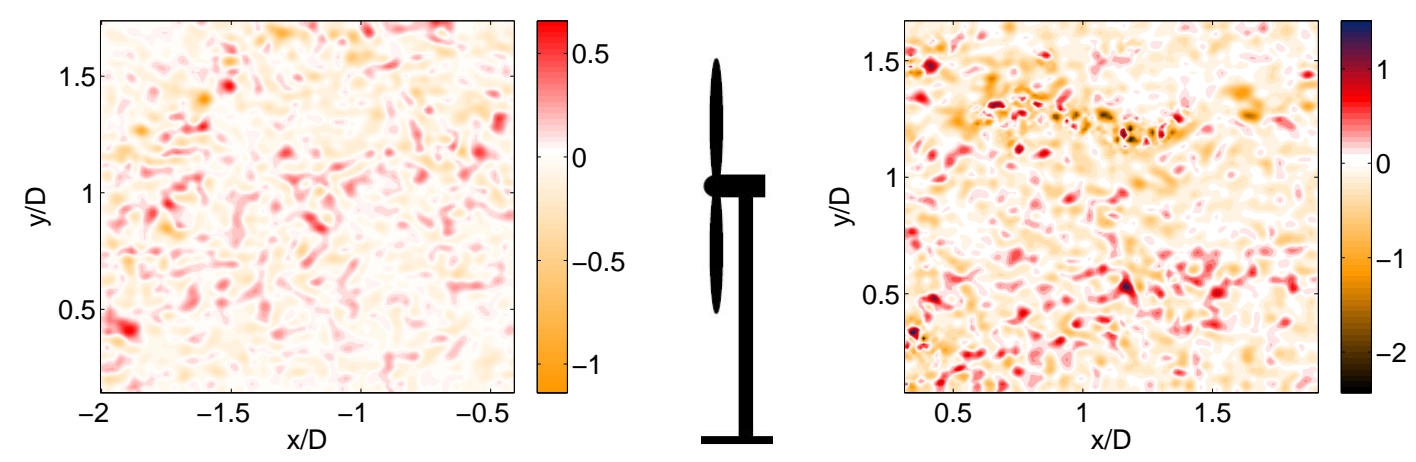

Figure 4.5: Vorticity, $\omega$.

The vortices at the top tip contain both positive (counter clock-wise rotation) and 
negative (clock-wise rotation) vorticity, whereas the bottom tip contains mainly positive vorticity. Close to the mast, in the wake, positive and negative vorticity can be seen. In front of the turbine, the regions of relatively low vorticity are distributed throughout the flow field. The vorticity magnitude in front of the turbine is less than half of that in the wake, indicating weakening vortices with advection downstream. Peak regions of vorticity are consistent with $\tilde{k}$ in Figure 4.4 .

The swirling strength is shown in Figure 4.6, where a non-zero $\lambda_{c i}^{2}$ represents a vortex. The sign of vorticity was referenced in the algorithm to display direction of rotation along with the swirling strength. As introduced by Zhou et al. [35], $\lambda_{c i}$ was squared to limit the level of background noise. The presence of the vortices are evident exactly in the shear layer region near the top tip. This is not as clear at the bottom tip due to the effects of the mast. The vortices are distributed throughout the contour in the front of the turbine as opposed to the wake. The peak strength of the vortices are smaller in magnitude by $25 \%$ in the front versus the wake.
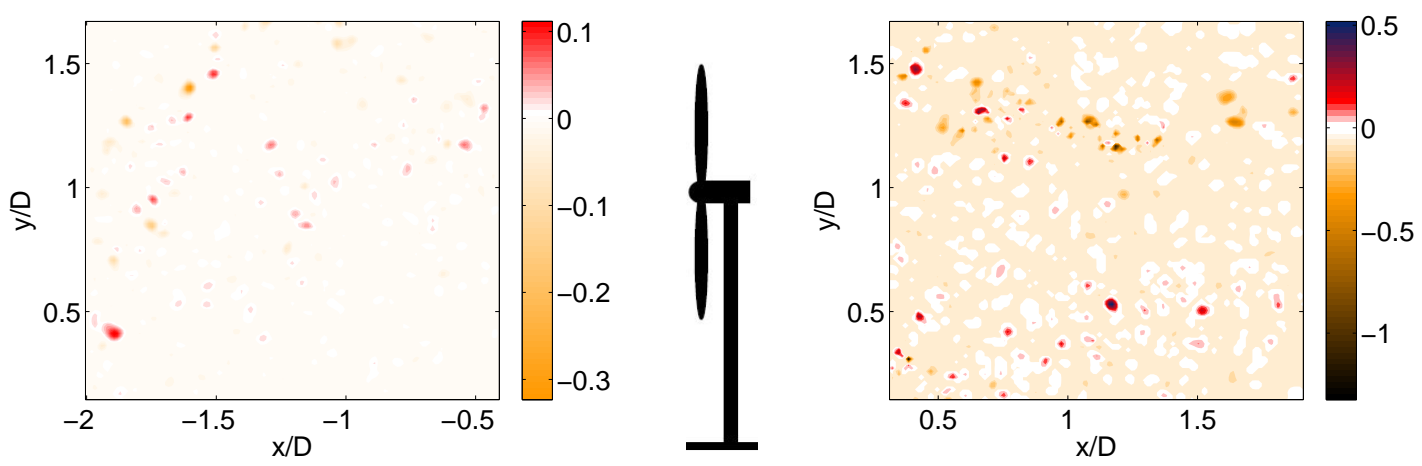

Figure 4.6: Swirling strength, $\lambda_{c i}^{2}$.

The direction of vortex rotation is distributed in front of the turbine while the top 
tip region in the wake is primarily positive and the bottom tip region primarily negative. This is a clear indication of vortices shedding from the blade tips.

Vortical regions are shown utilizing the Q-criterion in Figure 4.7, for $\mathrm{Q}>0$. Similar regions are identified as in the swirling strength (Figure 4.6). The non-zero regions in front of the turbine are more distributed throughout the field than in the wake. In the wake region, a large quantity of high magnitude vortices are shown at the top blade tip height. A few structures are visualized at the bottom tip and near the mast region in the wake. The majority of non-zero structures are of low magnitude, where they are located in the vicinity of peak regions. Low magnitude structures are also identified inside the wake of the turbine rotor. The resulting peak magnitude differences, for the wake compared to the front of the turbine, are approximately 4 times greater (as seen in Figure 4.6).
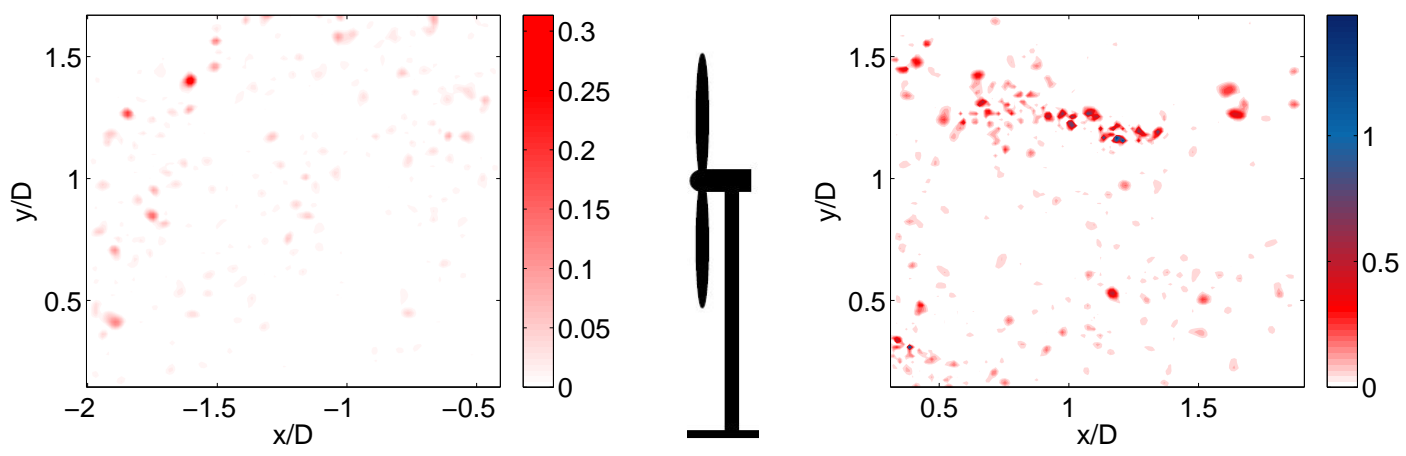

Figure 4.7: Q-criterion.

The vortical regions identified using the $\Delta$-criterion are shown in Figure 4.8, for $\Delta>0$. When comparing to the Q-criterion and swirling strength, the vortical regions are fewer in quantity. The vortical regions visible using the Q-criterion and 
not visible using the $\Delta$-criterion are of small magnitude. The results suggest that the $\Delta$-criterion is unable to detect vortices of small magnitude. This could be argued by the formulation (Equation 1.18), where $\mathrm{Q}$ and R, invariants of the VGT, are divided and the exponent is taken from this value resulting in the removal or dampening of the already small magnitude vortices.
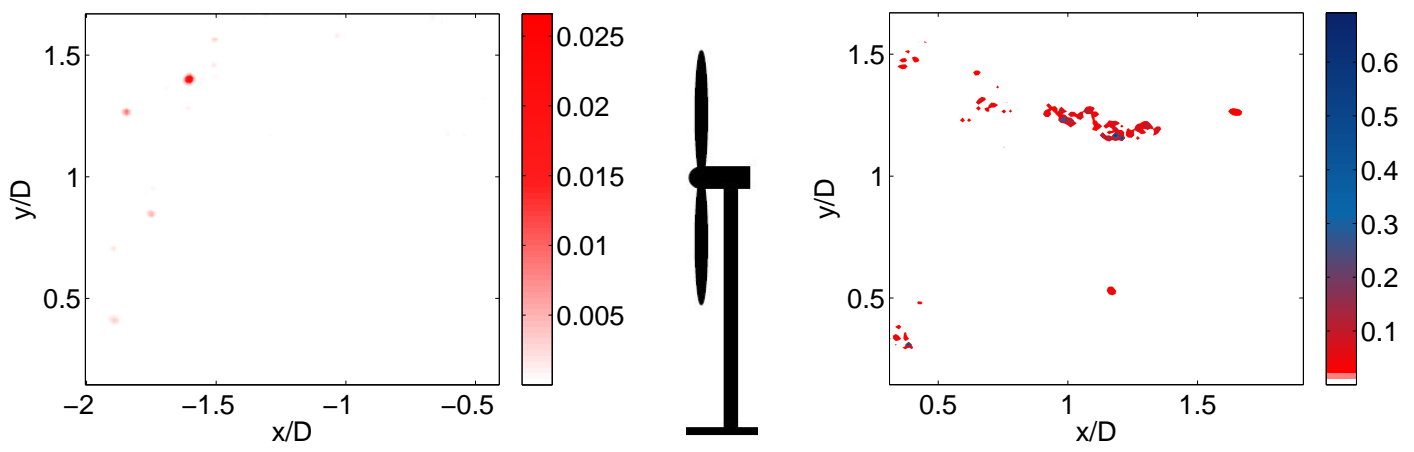

Figure 4.8: $\Delta$-criterion.

In Figure 4.9, the vortices identified using the $\lambda_{2}$-criterion are found to be distributed in front of the turbine and concentrated at the blade tip heights in the wake.
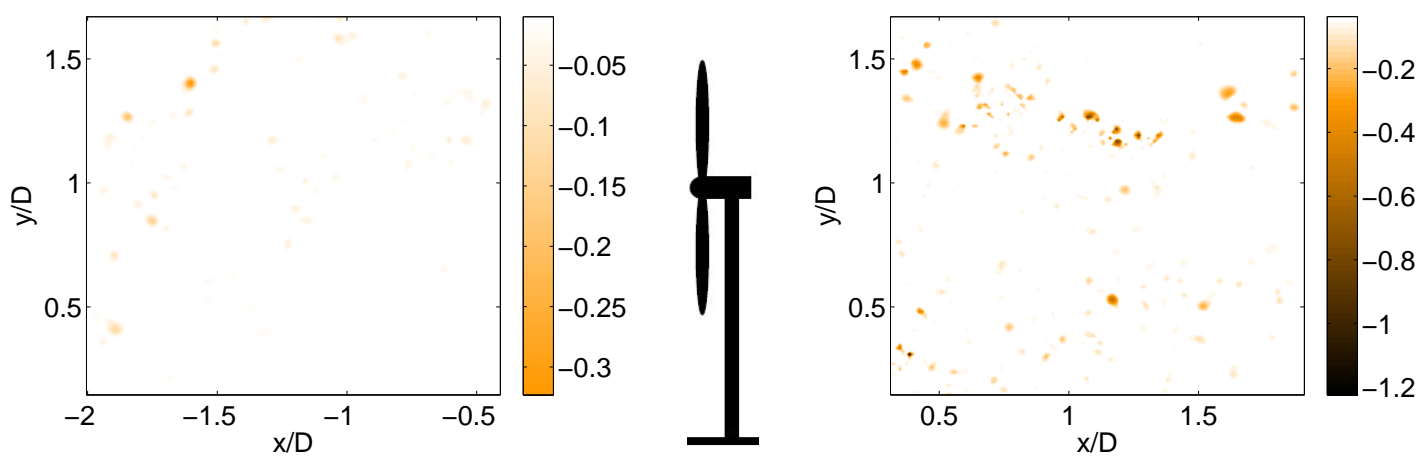

Figure 4.9: $\lambda_{2}$-criterion. 
The high magnitude vortices are visible to be the effects of the tip of the blades. The resulting vortices using the $\lambda_{2}$-criterion are similar to the Q-criterion in Figure 4.7. Similar peak magnitudes are resulted in Q-criterion and $\lambda_{2}$-criterion, where $\lambda_{2}$-criterion has negated peak values of Q-criterion. This result is in agreement with the argument posed by Jeong and Hussain [25] for planar flows. The main difference between the Q-criterion and $\lambda_{2}$-criterion is the resulting size of the identified structures. The $\lambda_{2}$-criterion generates a smaller vortex region than the Q-criterion, arguably a clearer indication of the vortex core.

The Galilean decomposition vector fields (shown using black vectors) at two percentages, 20 and 50, of the convection velocity $\left(U_{c}=7 \mathrm{~m} / \mathrm{s}\right)$ are shown in Figures 4.10 and 4.11, respectively. The plots are overlaid with the non-oriented swirling strength to provide a reference of the vector field to regions of vortices. The swirling strength colorbar magnitudes in Figures 4.10 and 4.11 are adjusted for increased clarity of vortex regions.
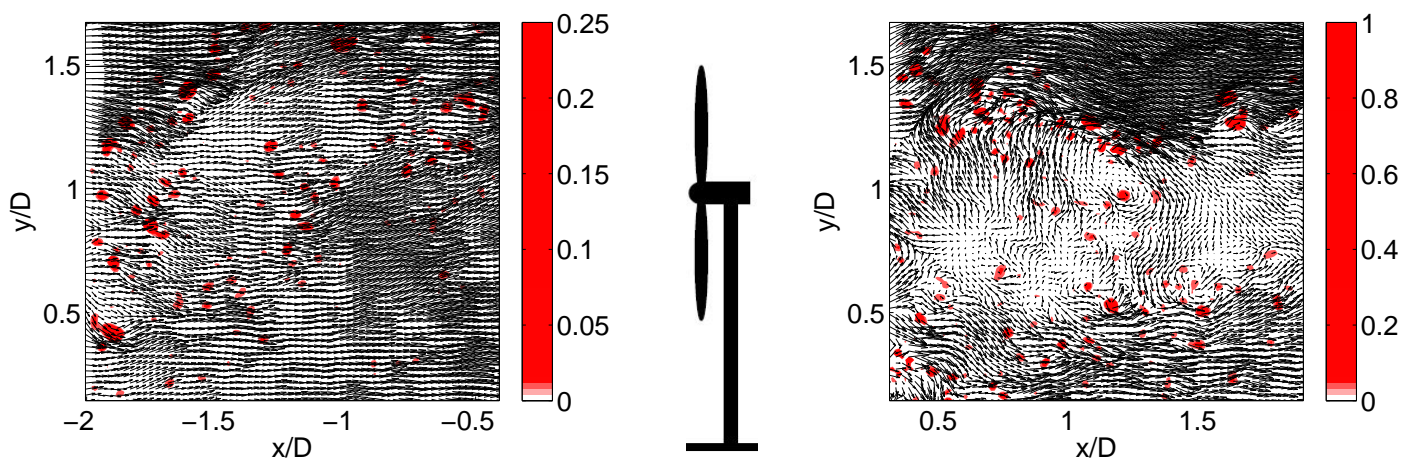

Figure 4.10: Galilean decomposition at $20 \%$ overlaid with non-oriented swirling strength. 
At 20 percent decomposition, the bulk flow entrainment is shown in the region of the top and bottom tip where the high speed flow interacts with the low speed flow in the wake (velocity deficit region). The wake outline and meandering is visualized and the high swirling strength vortices are shown to encapsulate the outer edge of the wake inside the shear layer. Inside the wake region, low magnitude swirling motion and velocity minima are resulted. The front region, at 20 percent decomposition, reveals no clear swirling motion indicating a requirement of further (increased percentage) decomposition. At 50 percent decomposition, the flow outside the wake and the front of turbine region is shown to contain swirling motion. The region above the wake reveals high magnitude vortices convecting with the entraining flow. Near the wall, the low magnitude swirling motions are visible. In the front of turbine region, there is a clear indication of swirling motion in agreement with the positive swirling strength. Although common vortices are visualized between either decompositions, when the proper convective velocity (i.e. decomposition percentage) is found, the vortices are shown to be clear and in agreement with the swirling strength.
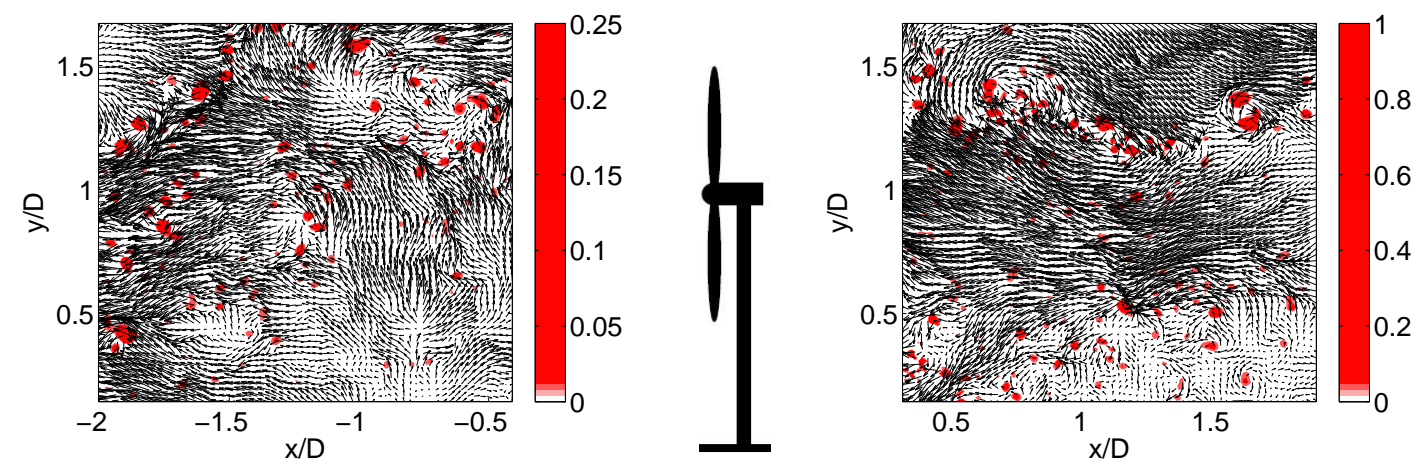

Figure 4.11: Galilean decomposition at 50\% overlaid with non-oriented swirling strength. 
The Reynolds decomposition vector fields are shown in Figure 4.12 overlaid with the swirling strength, as shown in Figure 4.10 and 4.11. The Reynolds decomposition shows an increased quantity of rotational motion when compared to one Galilean decomposition. Most regions of swirling motion are visible and in agreement with the positive swirling strength. Swirling motions are seen within the shear layer, at the top and bottom tip height, and outside the wake region. The flow upstream of the turbine shows swirling motion distributed throughout the plane highlighted by swirling strength high and low magnitude structures. The key difference between the Reynolds and Galilean decomposition is the visibility of the bulk flow behavior. The Reynolds decomposition is effective in uncovering the most swirling motions due to most vortices travelling with the local mean convective velocity, but the general flow behavior is eliminated. Reynolds decomposition is unable to clearly delineate the wake shape and the regions of flow entrainment where the high and low speed fluid interacts to generate a shear layer.
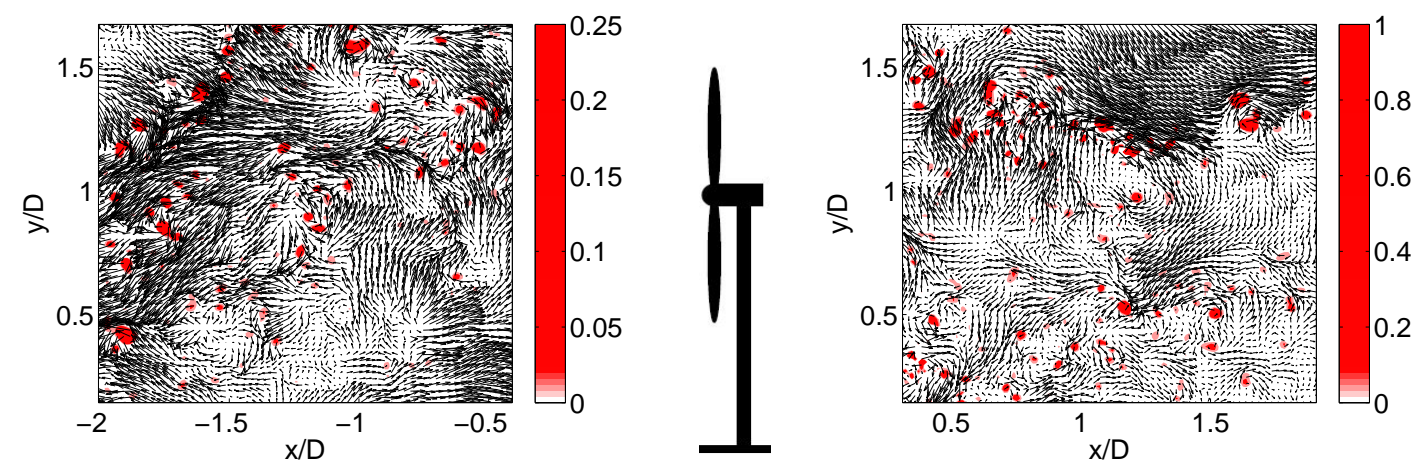

Figure 4.12: Reynolds decomposition (showing $u^{\prime}$ ) overlaid with non-oriented swirling strength.

The swirling strength peaks are hidden in the Reynolds decomposition vector plot 
at the top tip wake regions as well as directly in front of the turbine near hub height, indicating a few vortices not travelling at the local mean velocity for the selected frame.

\subsection{Discussion}

In a wind farm, the incoming flow is found to collide at high speed with the turbine structure and generate a high velocity deficit. For 6D streamwise and 3D spanwise turbine spacing, the velocity deficit extends to the front of the following turbine, indicating that the flow does not completely recover. The incoming flow interacts with the blades, generating aerodynamic lift, resulting in increased angular velocity. The tips of the rotating blades shed vortices that are convected downstream. Entraining flow from above and below the turbine blades is visualized, this is the process of recovery of the lost flow momentum. During the process of flow momentum recovery, the high speed entraining fluid shears the low speed fluid inside the velocity deficit region. The maximum shearing magnitude is visible in the top tip region where the high magnitude tip vortices exist. The wake is observed to contain low and high magnitude vortices both close to the turbine and deep into the wake. High magnitude vortices are seen in the shear layer region where they are found to mix as they convect downstream, resulting in distributed vortical regions in front of the following turbine.

The vorticity plot allows identification of peak swirling motion in low shear regions but obscures the detail of vortices in the regions of high shear. The regions of peak vorticity are in agreement with the peak instantaneous turbulent kinetic 
energy, indicating a clear relationship with turbulence and vorticity.

The $\mathrm{Q}, \Delta, \lambda_{2}$, and swirling strength criteria clearly mark the vortices within the shear layer. Both high and low magnitude vortex regions are revealed utilizing Q, $\lambda_{2}$, and swirling strength criterion, whereas the $\Delta$-criterion is unable to reveal the low magnitude regions. The swirling strength criterion utilizes the method of identifying complex eigenvalues, similar to the $\Delta$-criterion. The important difference in the swirling strength criterion is in its focus on the imaginary portion of the complex conjugate. This enables it to capture all swirling motion regardless of magnitude, resulting in a superior method to the $\Delta$-criterion.

Ultimately, all the criteria utilized may suffer the same result of the $\Delta$-criterion if a single vortex or group of vortices of sufficiently high magnitude occur in a flow containing a majority of sufficiently low magnitude vortices. This fate cannot be avoided for frame dependent (Eulerian) criteria. The nature of the frame dependent criteria has sent some researches on a path to seek a more mathematically objective approach (i.e. Lagrangian). Although the reviewed criteria have potential weaknesses, the resources necessary to accomplish adequate results in the wake of a wind turbine array have proven the identification methods as effective.

Commonly, thresholds are utilized for clear demarcation of vortex cores. The techniques in this study resulted in regions of vortical motion with the use of zero value thresholds (not to be confused with the thresholds of convective velocities applied in the Galilean decomposition). As seen in the results, the regions of vortical 
activity were similar among the criterion, but the vortex sizes varied. Further work can be completed to capture and determine the appropriate size of the vortex. This can be accomplished by coupling the decomposition techniques with the identification criteria while adjusting non-zero threshold values.

Calculation of the vorticity simultaneously with any of the discussed criteria will enable directional reference in the results. This method was utilized in the swirling strength. The computational time increase resulting from the simultaneous calculation was minimal (refer to Appendix B for the calculation time comparison). The complexity of each algorithm varied based on the criteria (see Appendix A for the Matlab code for each criteria). The varying complexity in each algorithm resulted in varying computational time to achieve results. Further code optimization can be completed to reduce the computational time for each technique. 


\section{Chapter 5}

\section{Conclusions}

In this study, the flow in the near and far wake, within the region of the infinite wind turbine array, was analyzed. The regions of interest were interrogated utilizing vortex identification techniques to reveal vortex concentration zones as well as their strength and behavior as they convect downstream. Studies of infinite arrays such as these are important to ascertain proper placement in large wind farms and the associated loading conditions that are experienced.

Regions of peak turbulent kinetic energy were related to vorticity, swirling strength, Q-criterion, $\Delta$-criterion, and $\lambda_{2}$-criterion. The vortices shed from the blade tips were identified in the near wake where they concentrate in the shear layer at the edge of the wake region. Swirling strength, Q-criterion, $\Delta$-criterion, and $\lambda_{2}$-criterion clearly identified the details of vortices within the shear layer. The vortices meander about the wake region where they mix as they move downstream resulting in distributed vortex regions in the far wake. These vortices, in the far wake, retain 25 percent of their near wake strength before they collide with the next turbine in the array.

Outside the shear layer and inside the wake region, the vortex strength is weak 
and only swirling strength, Q-criterion, and $\lambda_{2}$-criterion are effective at identifying vortex structures. $\Delta$-criterion, by the nature of its formulation, filters out vortices of low magnitude. The low magnitude regions can be argued to be insignificant in affecting design guidelines for design of the turbine structure. Nonetheless, the point of weakness remains by strictly focusing on the discriminant of the characteristic equation for the velocity gradient tensor.

The Q-criterion is found to be most effective in accurately identifying vortices in the lowest amount of computation time, followed by the swirling strength and $\lambda_{2}$-criterion (see Appendix B for a table of computation times for each technique).

The Galilean and Reynolds decomposition are compared with the swirling strength to place the identification techniques in context of the raw vector field data. After two Galilean decompositions and a Reynolds decomposition, all swirling motions are visualized in a vector plot and in agreement with peak regions resulting from the swirling strength criterion.

Further work in applying thresholds to each technique should be completed. Knowledge of appropriate threshold values allows for representation of true vortex sizes. The determination of an appropriate threshold for each technique will streamline the vortex identification process for this application.

Galilean vortex identification techniques applied to SPIV data successfully generated vortex regions comparable to those found in field studies (Pedersen and 
Antoniou [7]) and wind tunnel experiments (Zhang et al. [6]). Swirling strength, Q-criterion, and $\lambda_{2}$-criterion were found as the appropriate identification techniques for the wind energy application. Decomposition techniques provided additional information such as speed of vortex convection as well as vortex size. Utilization of these techniques assists in understanding potential energy extraction through vortex analysis. 


\section{Bibliography}

[1] Global Wind Energy Outlook 2012: Global Wind Power Market Could Triple by 2020, Global Wind Energy Council (2012).

[2] L. P. Chamorro, F. Porté-Agel, Turbulent flow Inside and Above a Wind Farm: A Wind-Tunnel Study, Energies (2011), pp. 1916-1937.

[3] K. Saranyasoontorn, L. Manuel, Low-dimensional representations of inflow turbulence and wind turbine response using proper orthogonal decomposition, ASME (2005), pp. 1-10.

[4] A. Rosen, Y. Sheinman, The power fluctuations of a wind turbine, J. Wind En. Ind. Aerodyn. 59 (1996), pp. 51-68.

[5] L. Van Binh, T. Ishihara, P. Van Phuc, Y. Fujino, A peak factor for nonGaussian response analysis of wind turbine tower, J. Wind Eng. Ind. Aerodyn. 96 (2008), pp. 2217-2227.

[6] W. Zhang, C. D. Markfort, and F. Porté-Agel, Near-wake flow structure downwind of a wind turbine in a turbulent boundary layer, Experiments in Fluids 52(5) (2012), pp. 1219-1235.

[7] T. F. Pedersen, I. Antoniou, Visualisation of flow through a stall-regulated wind turbine rotor, Wind Eng. 13 (1989), pp. 239-245.

[8] R. B. Cal, J. Lebron, L. Castillo, H. -S. Kang, C. Meneveau, Experimental study of the horizontally averaged flow structure in a model wind-turbine array boundary layer, Journal of Renewable and Sustainable Energy 2(013106) (2010), pp. $1-25$.

[9] N. Hamilton, H. -S. Kang, C. Meneveau, R. B. Cal, Statistical analysis of kinetic energy entrainment in a model wind turbine array boundary layer, Journal of Renewable and Sustainable Energy 4(6) (2012), pp. 063105-063105

[10] H. J. Lugt, 1983. Vortex Flow in Nature and Technology. Wiley 
[11] R. J. Adrian, K. T. Christensen, Z.-C. Liu, Analysis and interpretation of instantaneous turbulent velocity fields, Experiments in Fluids 29 (2000), pp. 275290.

[12] A. E. Perry, M. S. Chong, A series-expansion of the Navier-Stokes equations with applications to three-dimensional separation patterns, Journal of Fluid Mechanics 173 (1986), pp. 207-223.

[13] R. W. Metcalfe, F. Hussain, S. Menon, M. Hayakawa, Coherent structures in a turbulent mixing layer: a comparison between numerical simulations and experiments Turbulent Shear Flows 5 (1985), p. 110.

[14] A. K. M. F. Hussain, Coherent structures and turbulence, Journal of Fluid Mechanics 173 (1986), pp. 303-356.

[15] D. K. Bisset, R. A. Antonia, L. W. Browne, Spatial organization of large structures in the turbulent far wake of a cylinder, Journal of Fluid Mechanics 218 (1990), p. 439.

[16] J. Jiménez, A. A. Wray, P. G. Saffman, R. S. Rogallo, The structure of intense vorticity in isotropic turbulence, Journal of Fluid Mechanics 255 (1993), pp. 65-90.

[17] Y. Dubief, F. Delcayre, On coherent-vortex identification in turbulence, Journal of Turbulence 1(N11) (2000), pp 1-23.

[18] J. W. Brooke, T. J. Hanratty, Origin of turbulence producing eddies in a channel flow, Physics of Fluids 1011 (1993), pp. 1-13.

[19] S. K. Robinson, The kinematics of turbulent boundary layer structure, Ph. D. Thesis (1991)

[20] P. Comte, J. Silvestrini, P. Begou, Streamwise vortices in large eddy simulation of mixing layers, European Journal of Mechanics B/Fluids 17 (1998), pp. 615637.

[21] G. Haller, An objective definition of a vortex, Journal of Fluid Mechanics 525 (2005), pp. 1-26.

[22] J. C. R. Hunt, A. A. Wray, P. Moin, Eddies, Streams, and Convergence Zones in Turbulent Flows, Center for Turbulence Research: Proceedings of the Summer Program (1988), pp. 193-208. 
[23] A. Okubo, Horizontal dispersion of floatable particles in the vicinity of velocity singularities such as convergences, Deep-Sea Research 17 (1970), pp. 445-454.

[24] J. Weiss, The dynamics of enstrophy transfer in two-dimensional hydrodynamics, Physica D 48 (1991), pp. 273-294.

[25] J. Jeong, F. Hussain, On the identification of a vortex, Journal of Fluid Mechanics 285 (1995), pp. 69-94.

[26] R. Cucitore, M. Quadrio, A. Baron, On the effectiveness and limitations of local criteria for the identification of a vortex, European Journal Mechanics B/Fluids 18 (1999), pp. 261-282.

[27] K. Horiuti, A classification method of vortex sheet and tube structures in turbulent flows, Physics of Fluids 13 (2001), pp. 1-20.

[28] P. Chakraborty, S. Balanchandar, R. J. Adrian, On the relationships between local vortex identification schemes, Journal of Fluid Mechanics 535 (2005), pp. 189-214.

[29] S. J. Lawson, G. N. Barakos, Computational Fluid Dynamics Analyses of Flow over Weapons-Bay Geometries, Journal of Aircraft 47 (2010), pp. 1-19.

[30] H. Vollmers, H. P. Kreplin, H. U. Meier, Separation and vortical type flow around a prolate spheroid - evaluation of relevant parameters, Aerodynamics of vortical type flows in three dimensions; papers presented and discussions held at the Fluid Dynamics Panel Symposium at the Atlanta H. 342 (1983), pp. 14-1-14-14.

[31] U. Dallman, Topological Structures of Three-Dimensional Vortex Flow Separation, AIAA-83-1735 (1983), pp. 1-10.

[32] M. R. Spiegel and J. Liu, Schaum's Outlines: Mathematical Handbook of Formulas and Tables Second Edition, McGraw-Hill 1999.

[33] A. E. Perry, M. S. Chong, A Description of Eddying Motions and Flow Patterns Using Critical-Point Concepts, Annual Review of Fluid Mechanics 19 (1987), pp. 125-155.

[34] U. Dallman, A. Hilgenstock, S. Riedelbanh, B. Schulte-Werning, H. Vollmers, On the footprints of three dimensional separated vortex flows around blunt bodies, Vortex flow aerodynamics (1991), pp. 9-1-9-13. 
[35] J. Zhou, R. J. Adrian, S. Balachandar, T. M. Kendall, Mechanisms for generating coherent packets of hairpin vortices in channel flow, Journal of Fluid Mechanics 387 (1999), pp. 353-396.

[36] M. S. Chong, A. E. Perry, B. J. Cantwell, A general classification of threedimensional flow fields, Phys. Fluids A 2 (1990), pp. 765-777.

[37] N. Hamilton, M. Melius, R. B. Cal, Wind turbine boundary layer arrays for Cartesian and staggered configurations- Part I, flow field and power measurements, Wind Energy (2014).

[38] G. Strang, 18.06SC Linear Algebra, Fall 2011, MIT OpenCourseWare: Massachusetts Institute of Technology,

http://ocw.mit.edu/courses/mathematics/18-06sc-linear-algebra-fall -2011/positive-definite-matrices-and-applications/positive-definite -matrices-and-minima/MIT18_06SCF11_Ses3.3sum.pdf

(Accessed November 13, 2014). License: Creative commons BY-NC-SA 


\section{Appendix A}

\section{Vortex Identification Matlab Codes}

Appendix A contains Matlab codes for the vortex identification criteria utilized in this study: Vorticity, Swirling Strength, Q-criterion, $\Delta$-criterion, and $\lambda_{2}$-criterion.

\section{A.1 Vorticity $(\omega)$}

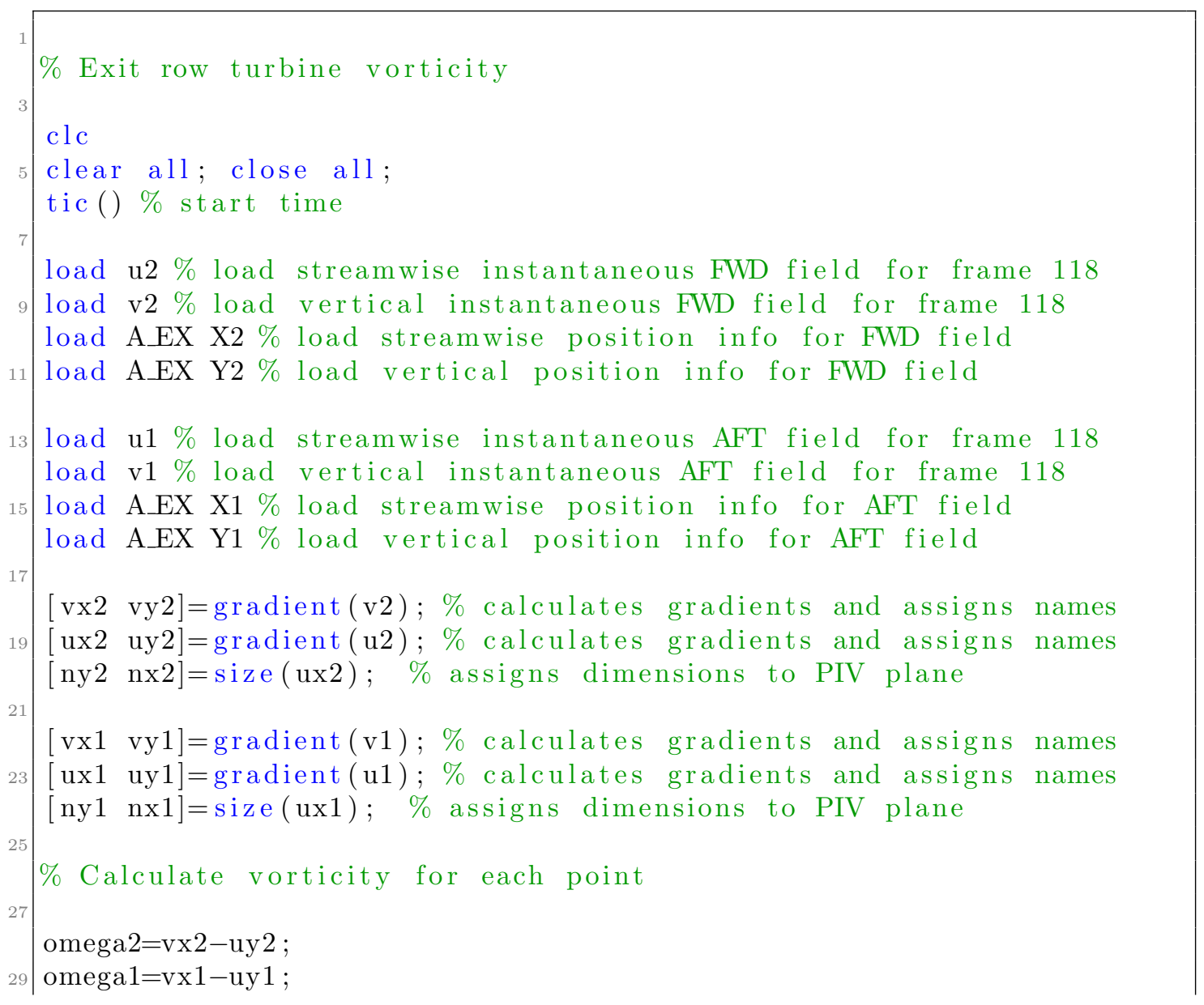




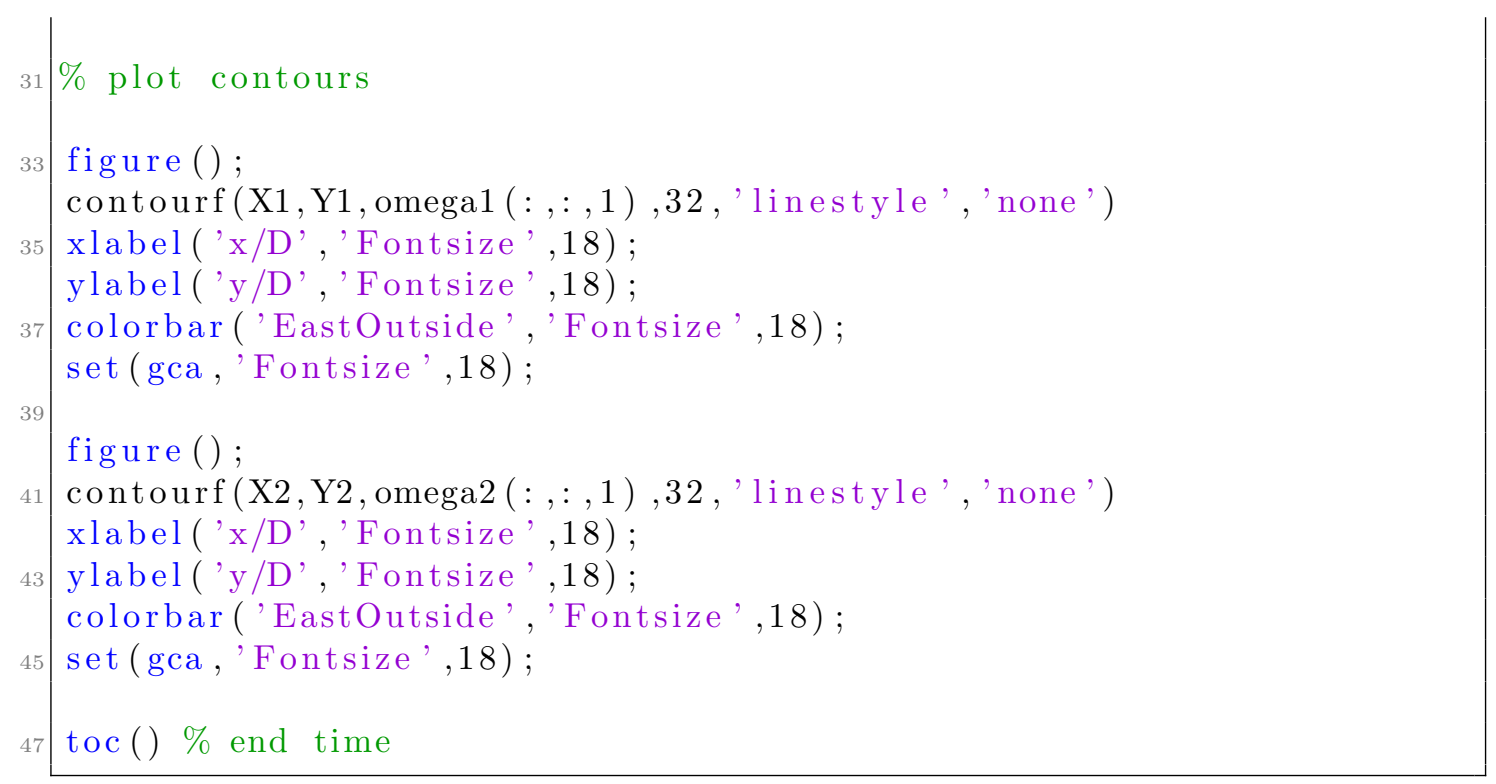

\section{A.2 Swirling Strength $\left(\lambda_{c i}^{2}\right)$}

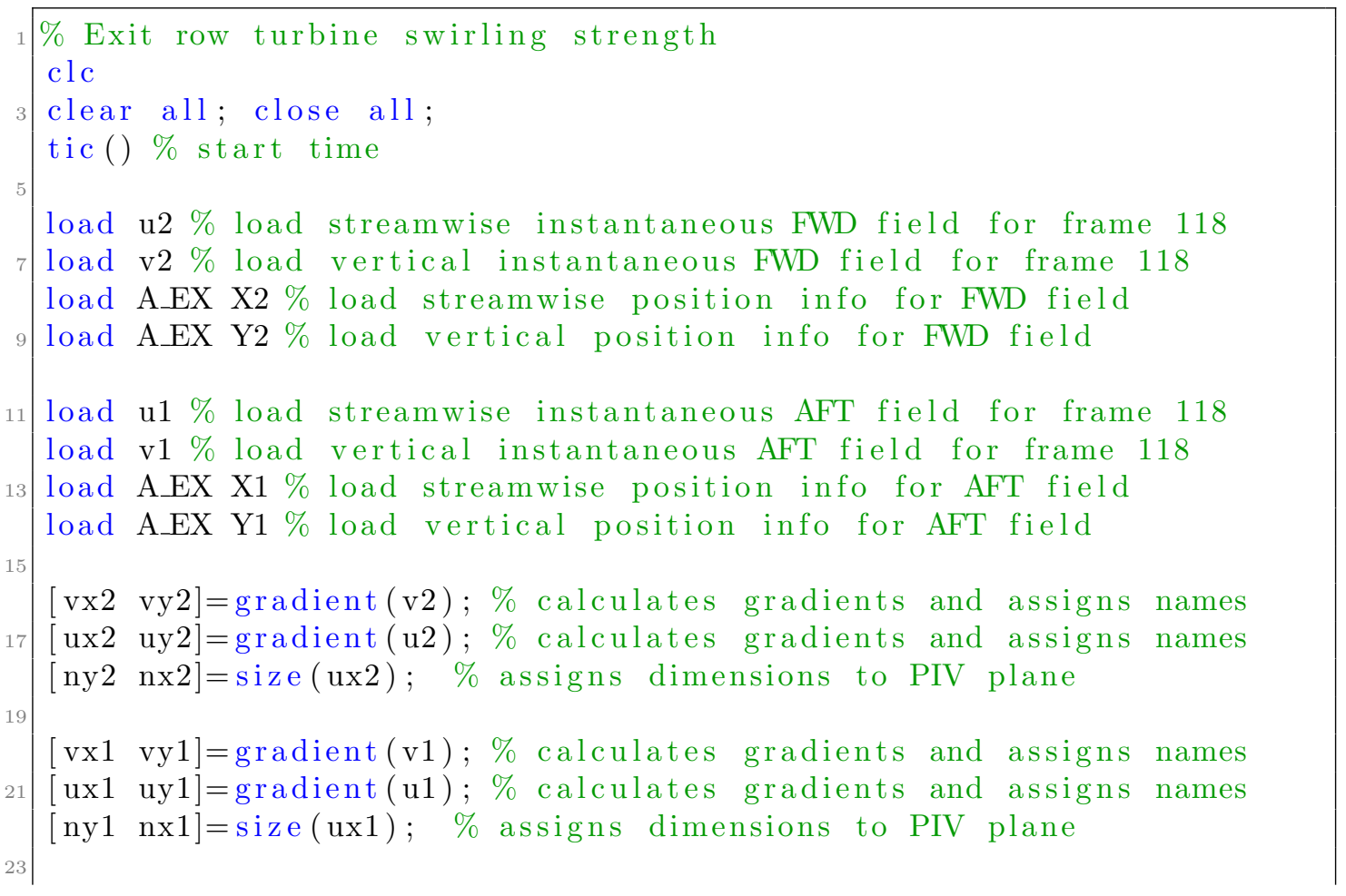




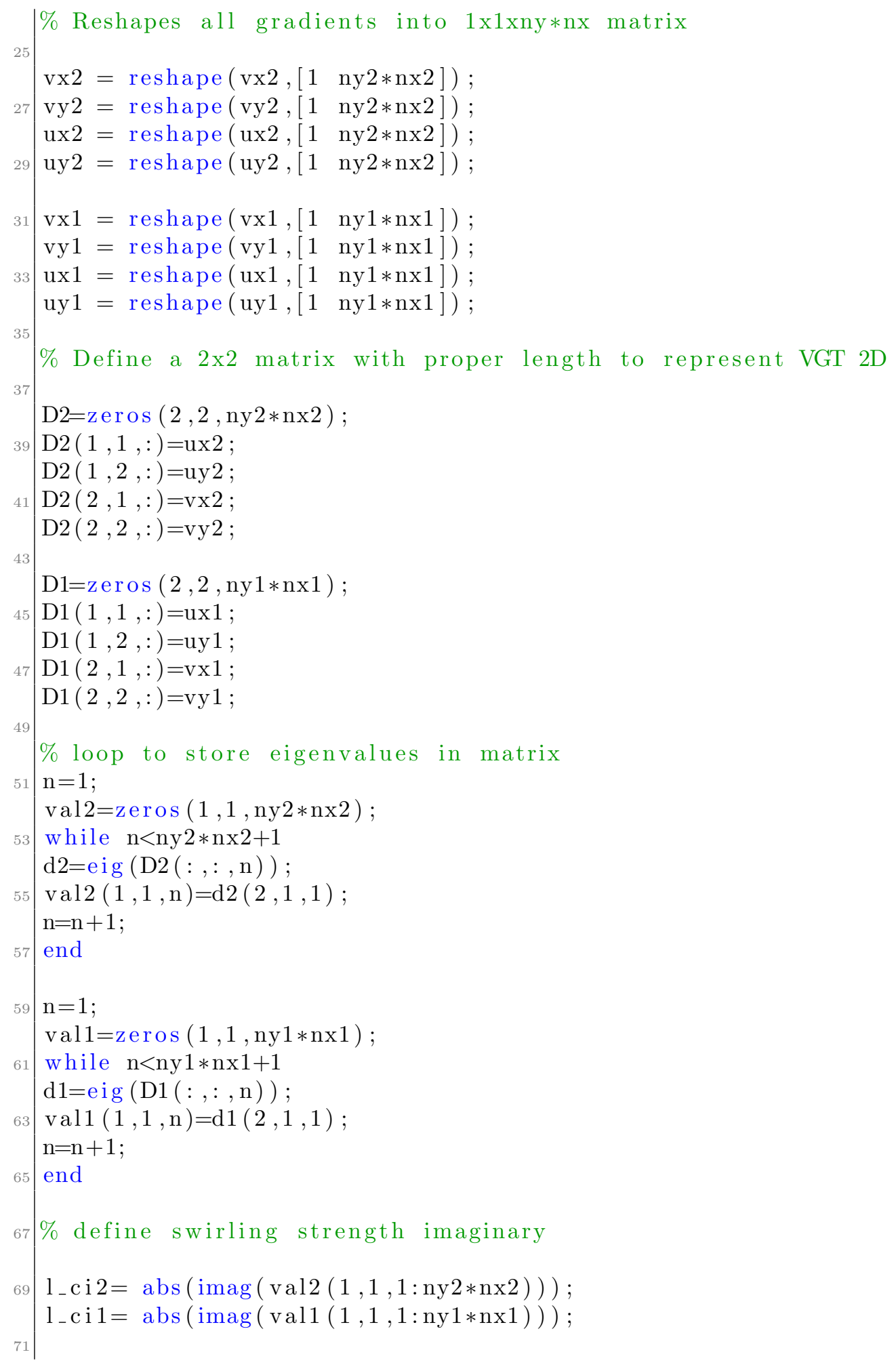




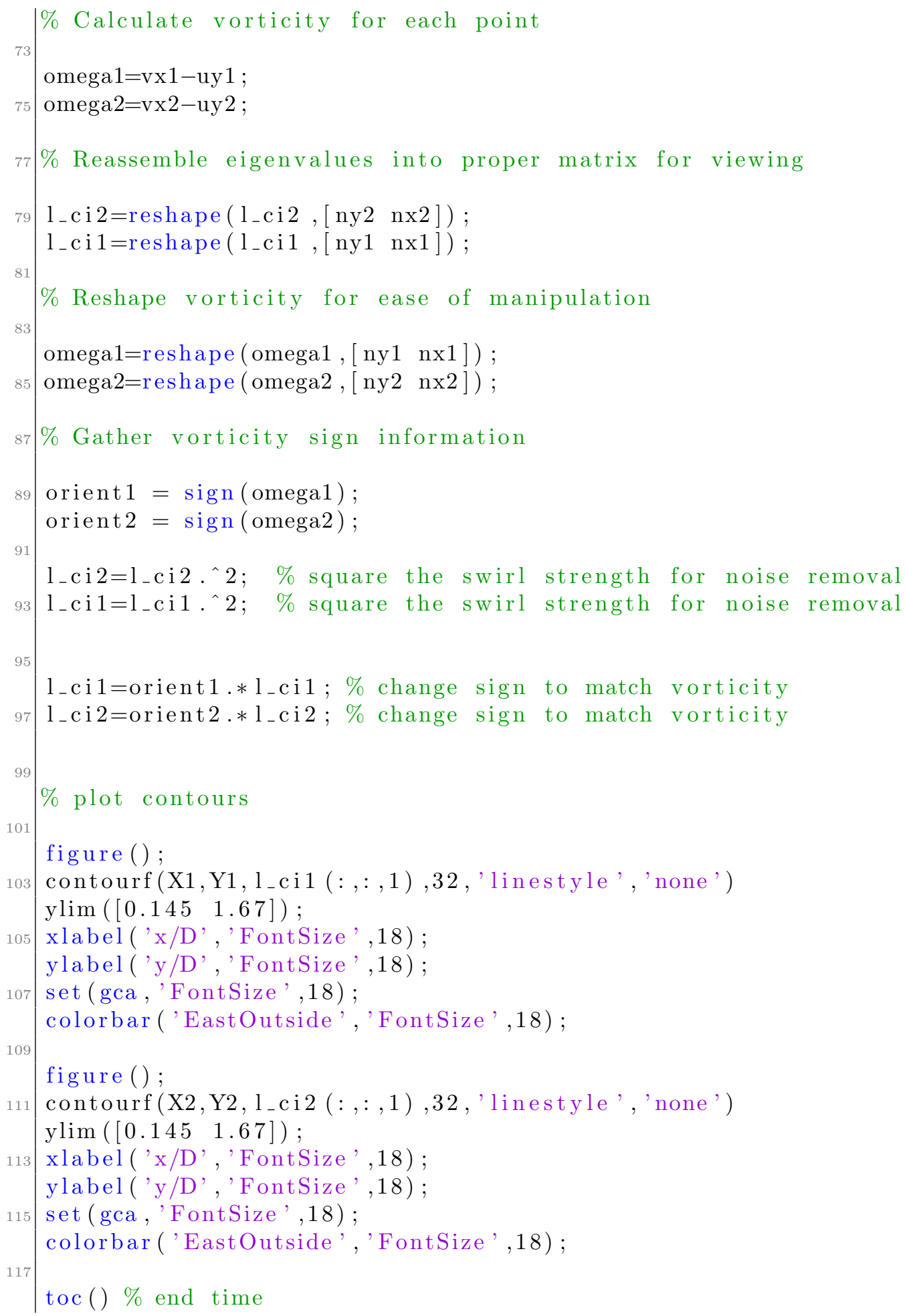




\section{A.3 Q-criterion}

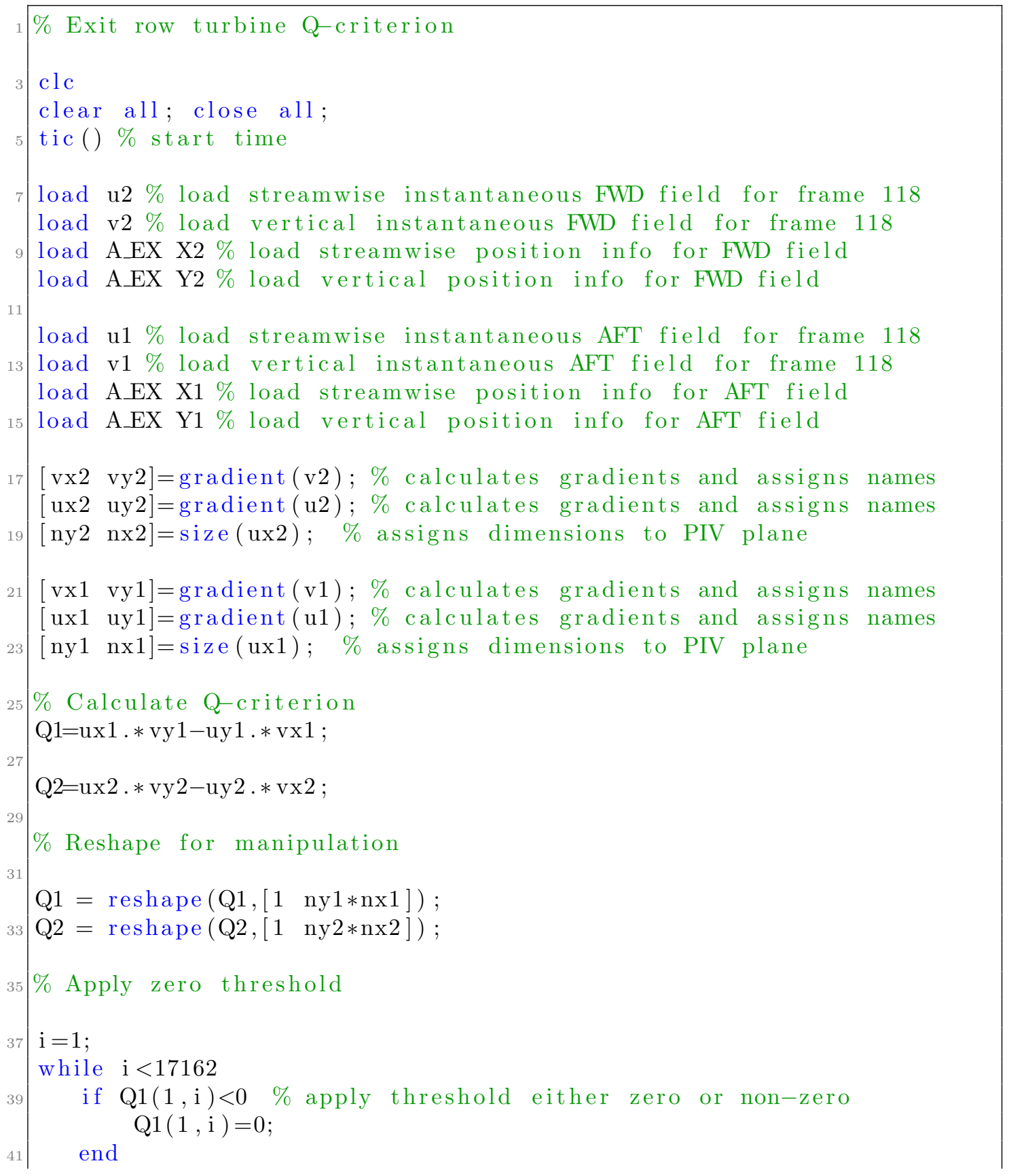




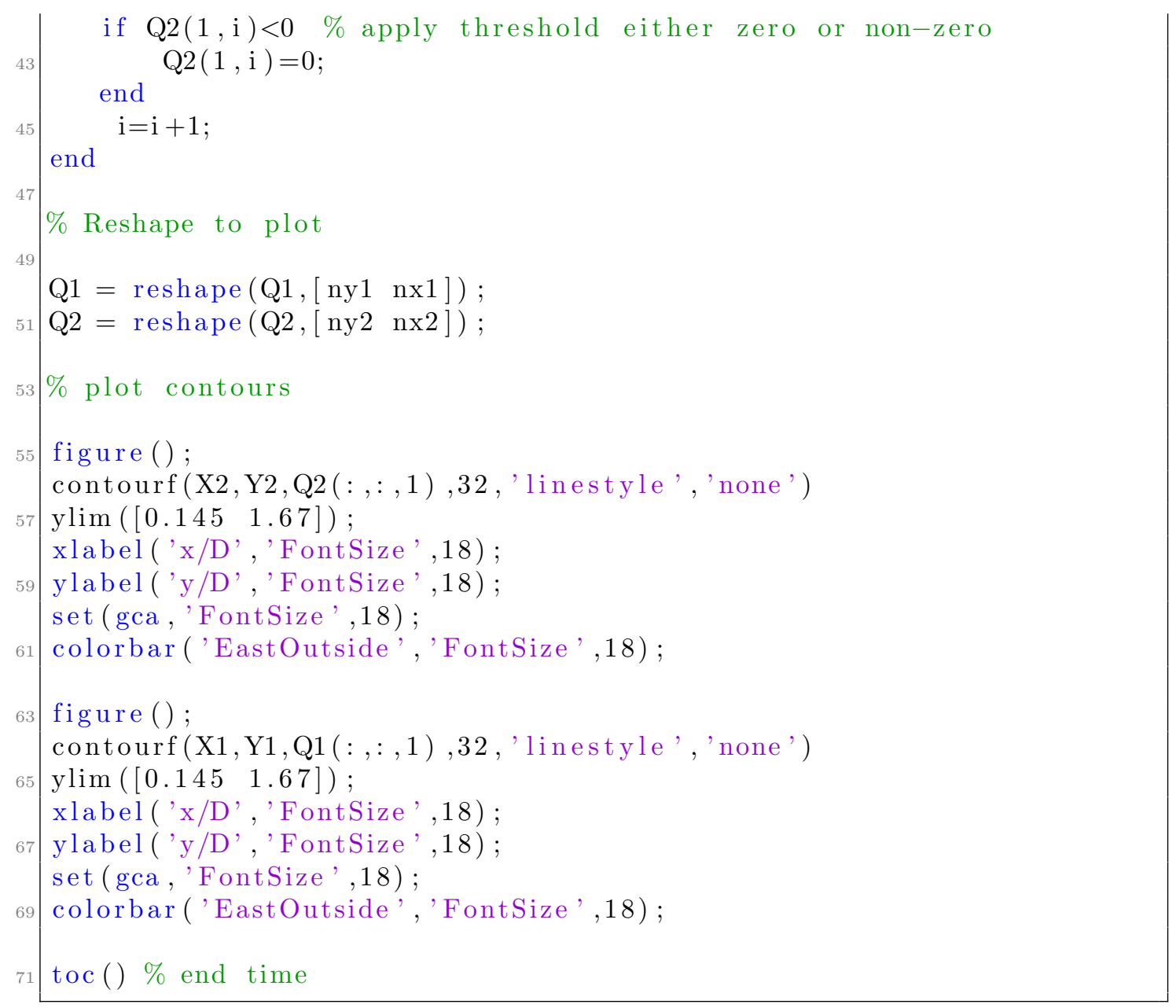

\section{A.4 $\Delta$-criterion}

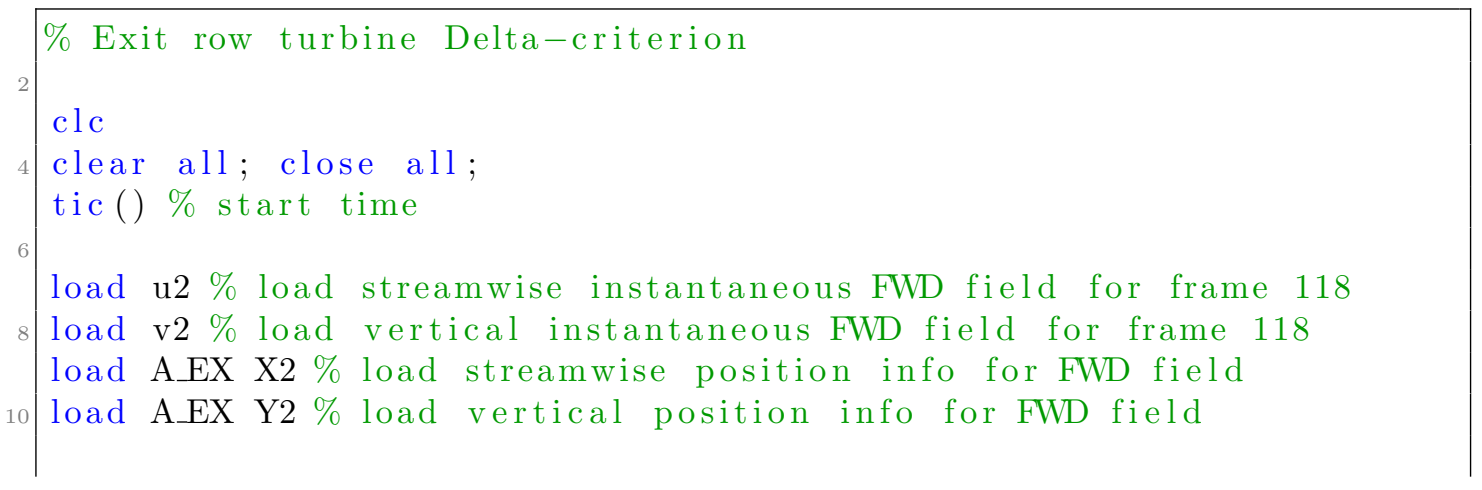




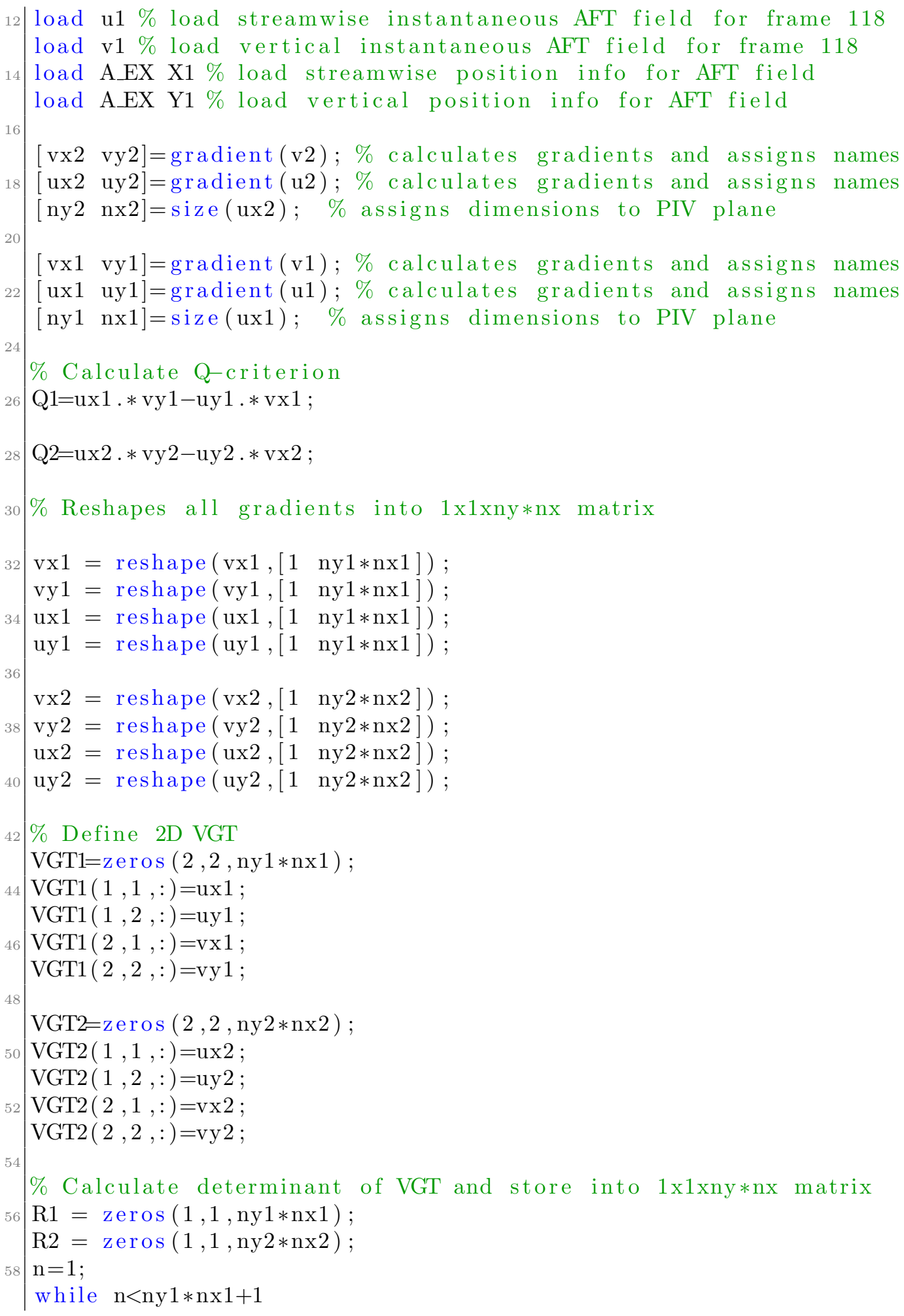




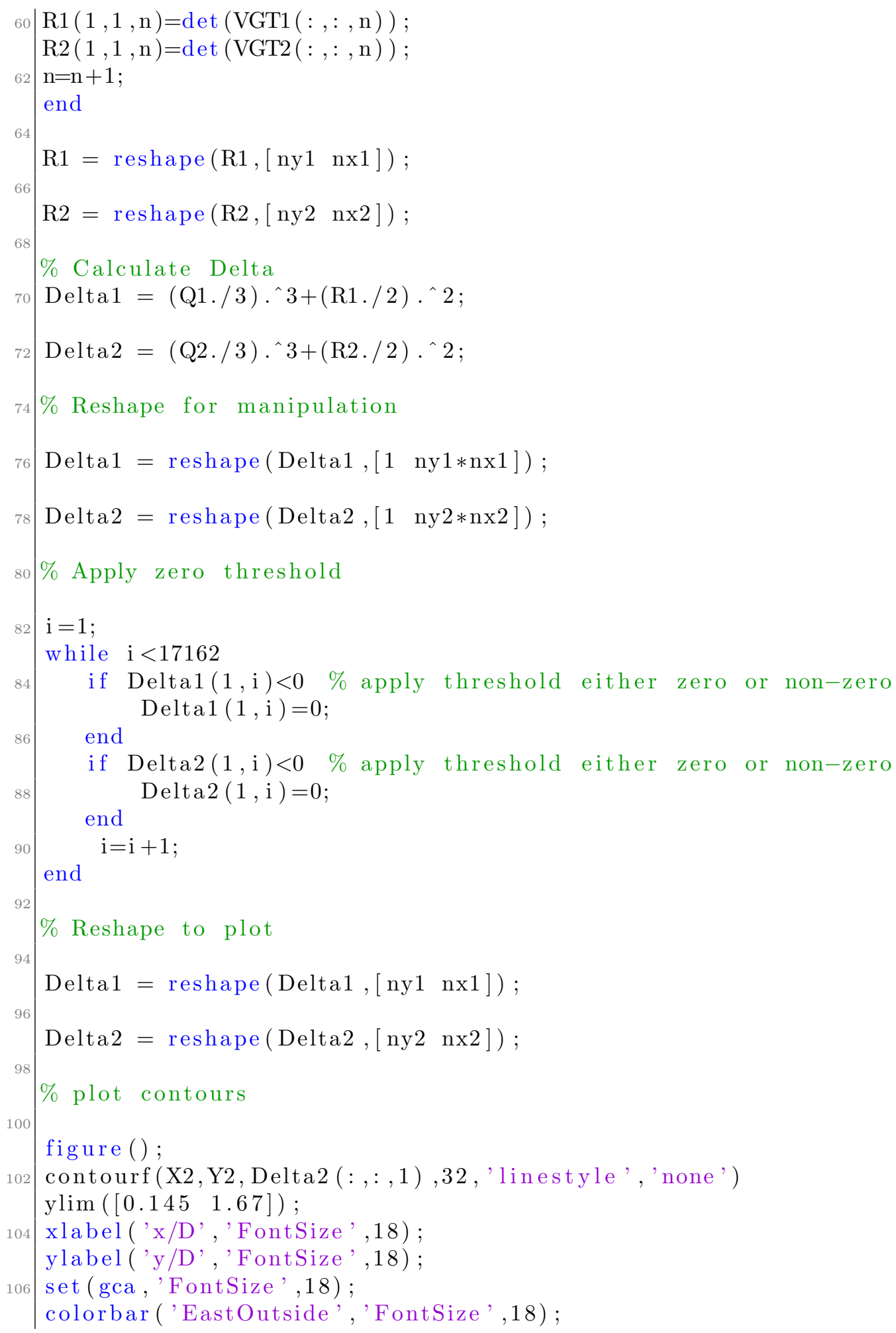




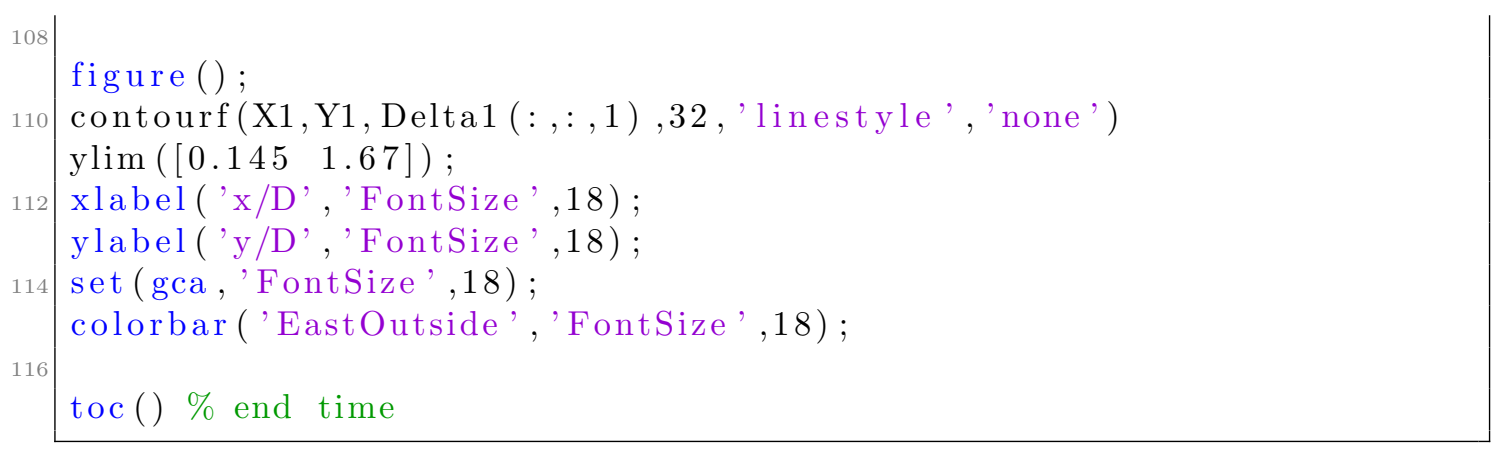

\section{A.5 $\lambda_{2}$-criterion}

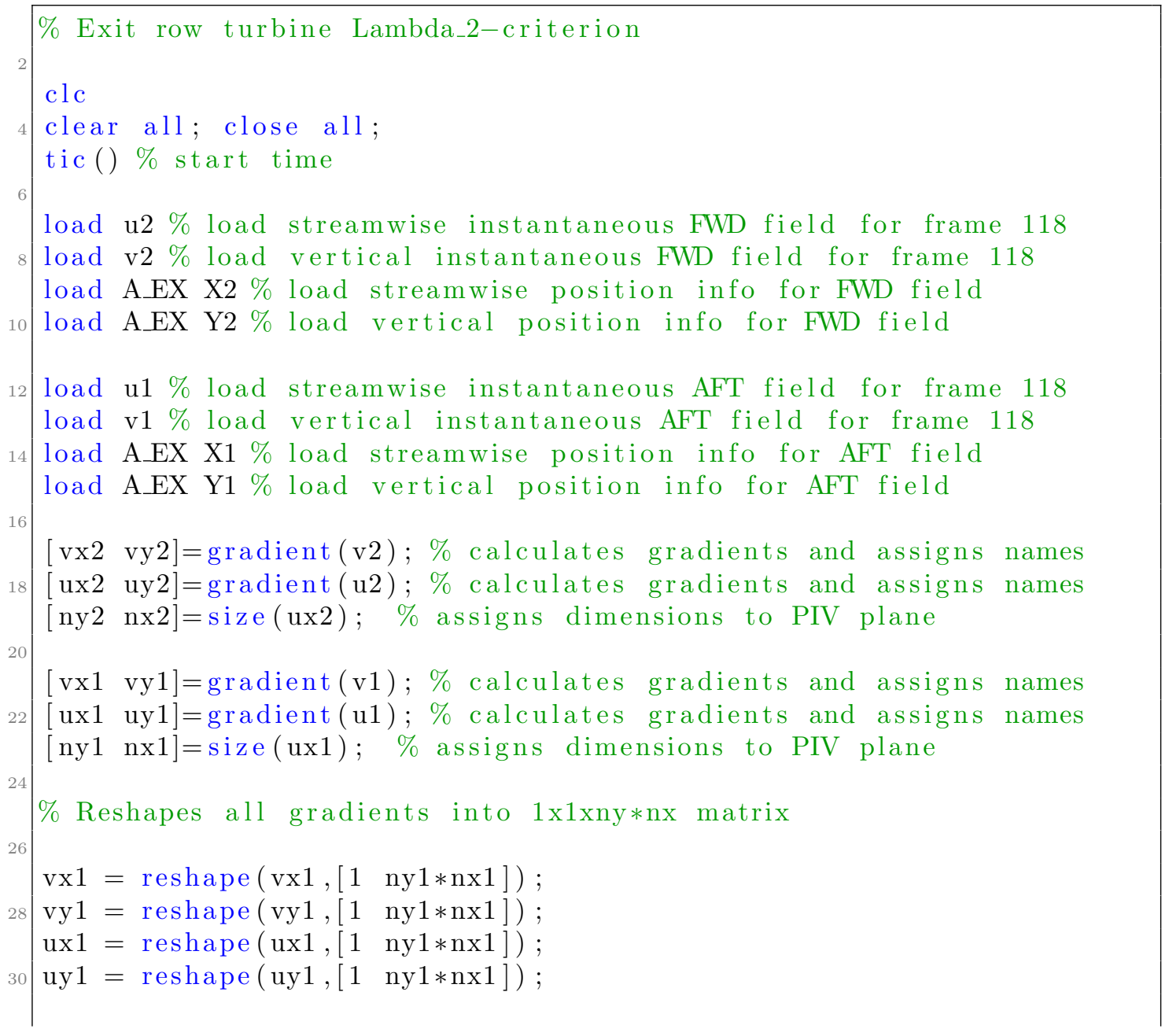




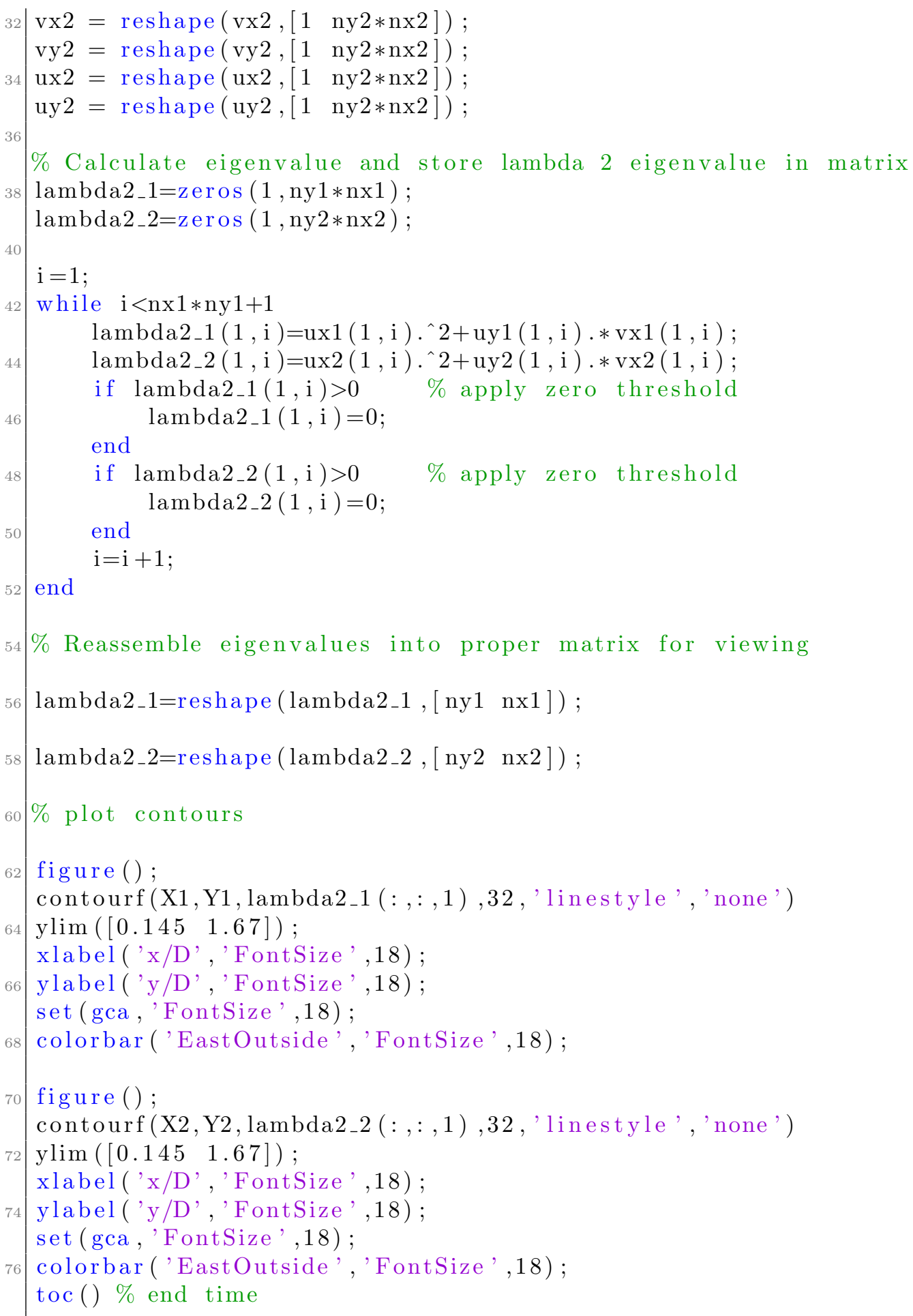




\section{Appendix B}

\section{Calculation Time for Each Criteria}

Table B.1 displays the calculation time for each technique used in the study. The average time was calculated based on 10 runs of each technique, where the resulting deviation of run time is shown as a percentage. The difference in the swirling strength oriented and non-oriented is the incorporation of the directional information resulting from the vorticity calculation.

Table B.1: Criteria run-time

\begin{tabular}{ccccccc}
\hline \hline Criteria & $\omega$ & $\lambda_{c i}^{2}$ (oriented) & $\lambda_{c i}^{2}$ & $\mathrm{Q}$ & $\Delta$ & $\lambda_{2}$ \\
\hline Average $(\mathrm{sec})$ & 5.88 & 4.10 & 3.88 & 3.60 & 2.84 & 5.09 \\
Deviation $( \pm \%)$ & 3 & 2 & 3 & 3 & 4 & 2 \\
\hline
\end{tabular}




\section{Appendix C}

\section{Frame Selection}

Figures $\mathrm{C} 1$ through $\mathrm{C} 5$ are consecutive frames displaying results of the swirling strength. The frames begin at 113 and continue to 117 (directly before the frame used in the study, 118). The magnitude colorbars have been adjusted based on the limits from frame 118.
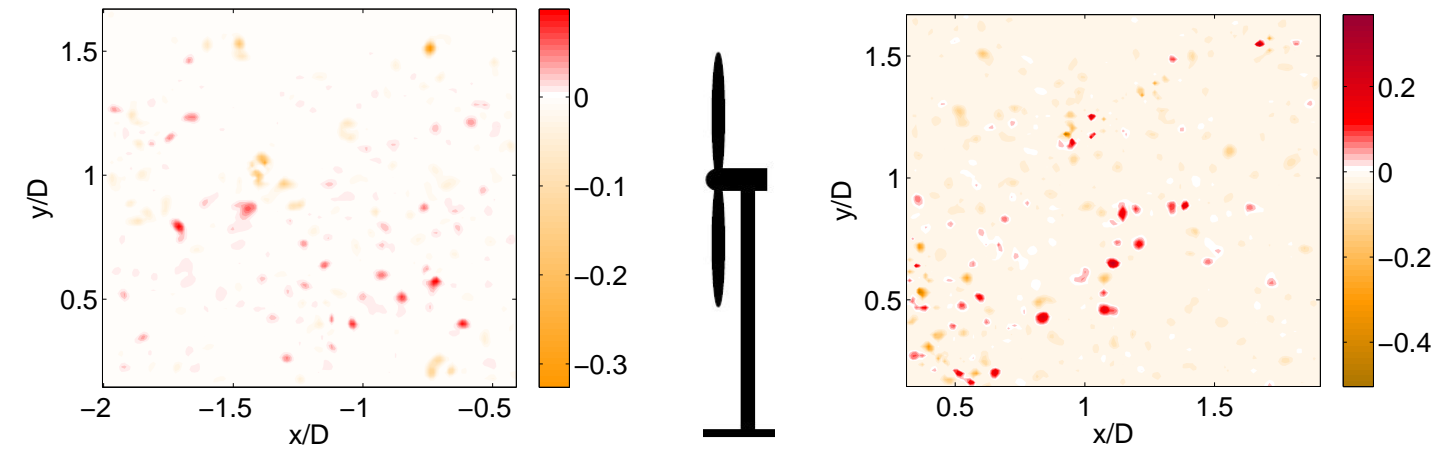

Figure C1: Swirling Strength $\left(\lambda_{c i}^{2}\right)$, Frame 113.
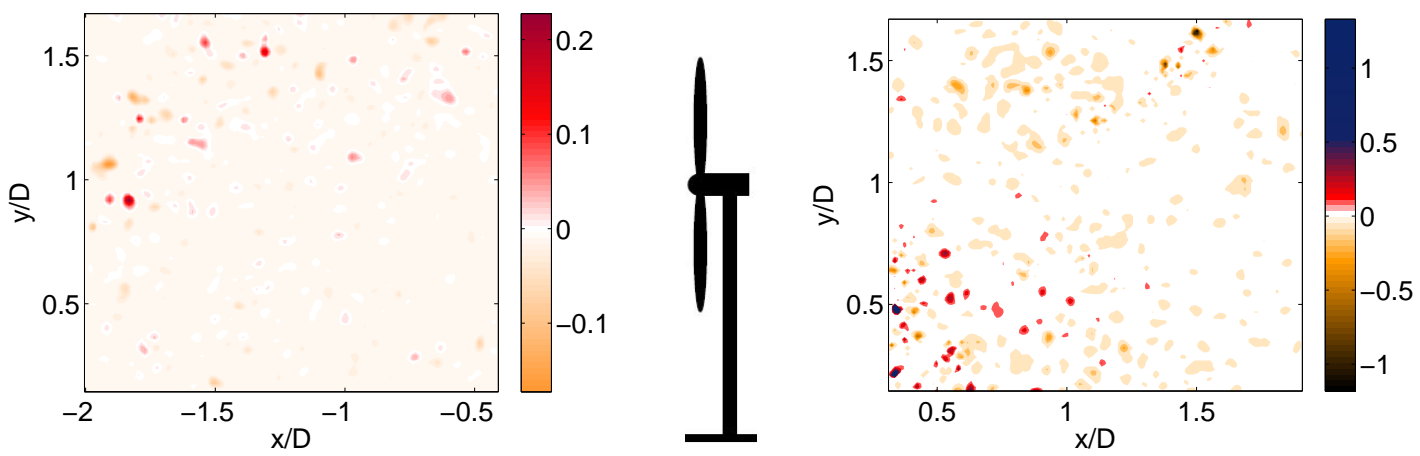

Figure C2: Swirling Strength $\left(\lambda_{c i}^{2}\right)$, Frame 114. 

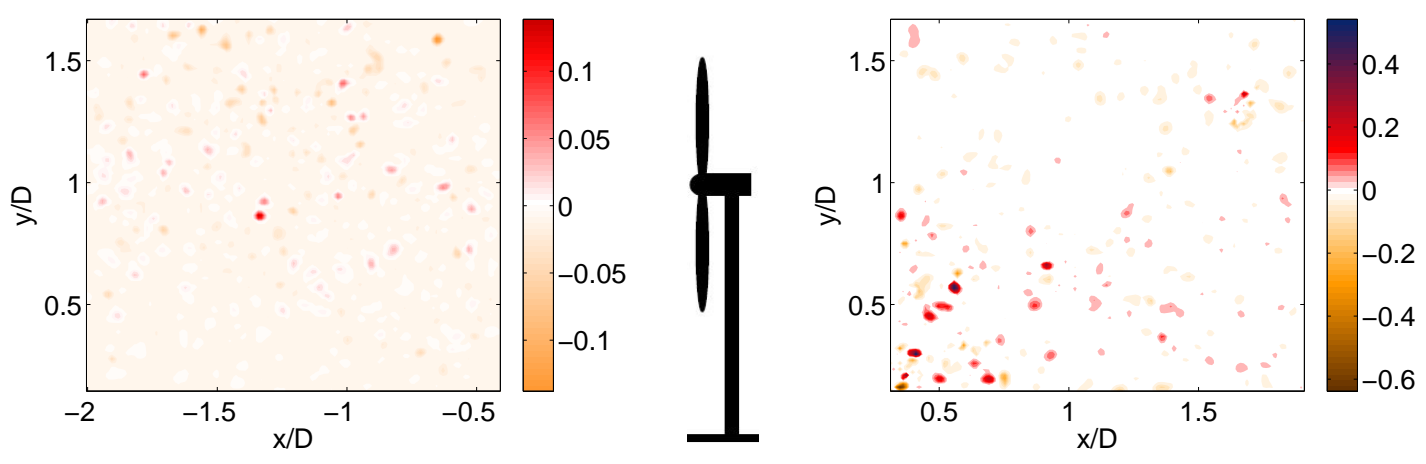

Figure C3: Swirling Strength $\left(\lambda_{c i}^{2}\right)$, Frame 115.
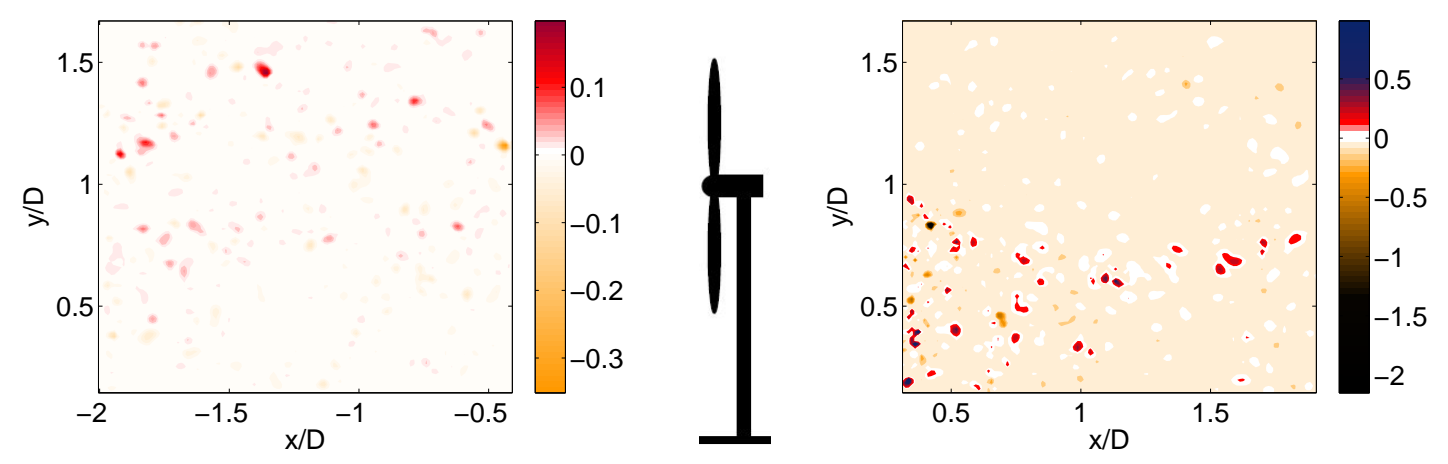

Figure C4: Swirling Strength $\left(\lambda_{c i}^{2}\right)$, Frame 116.
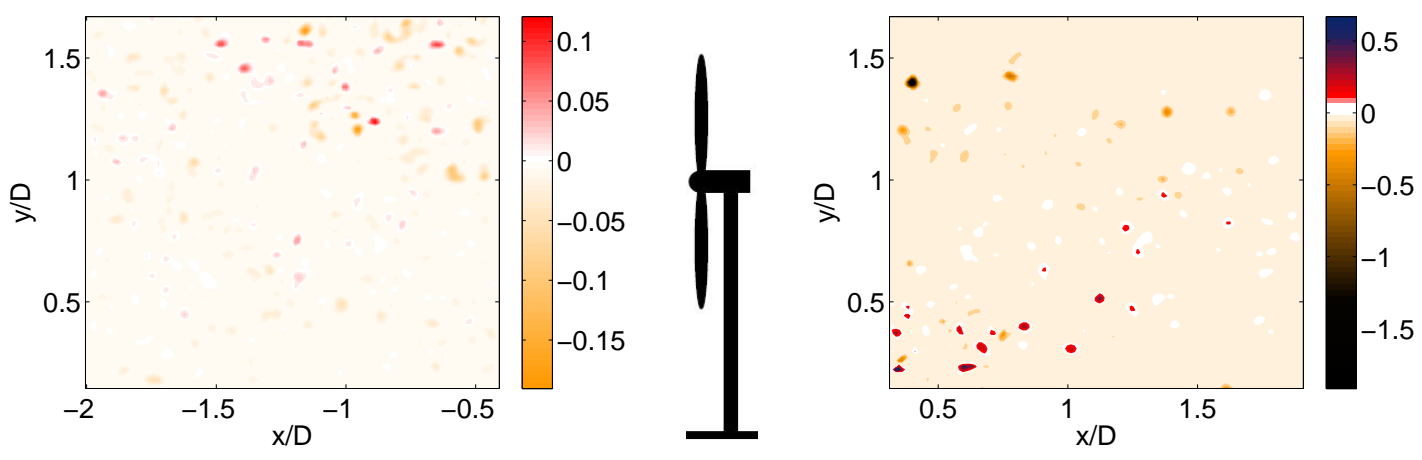

Figure C5: Swirling Strength $\left(\lambda_{c i}^{2}\right)$, Frame 117. 


\section{Appendix D}

\section{Threshold Selection for Front and Back Frame of Turbine}

Figure D1 is the $\lambda_{2}$-criterion frame used in the study. The magnitudes have been adjusted in the front frame to match that of the back frame. The resulting vortex regions in the front of the turbine are not easily visualized. This lead to the idea of one color for the peak value of each region, front and back (as used in the study).
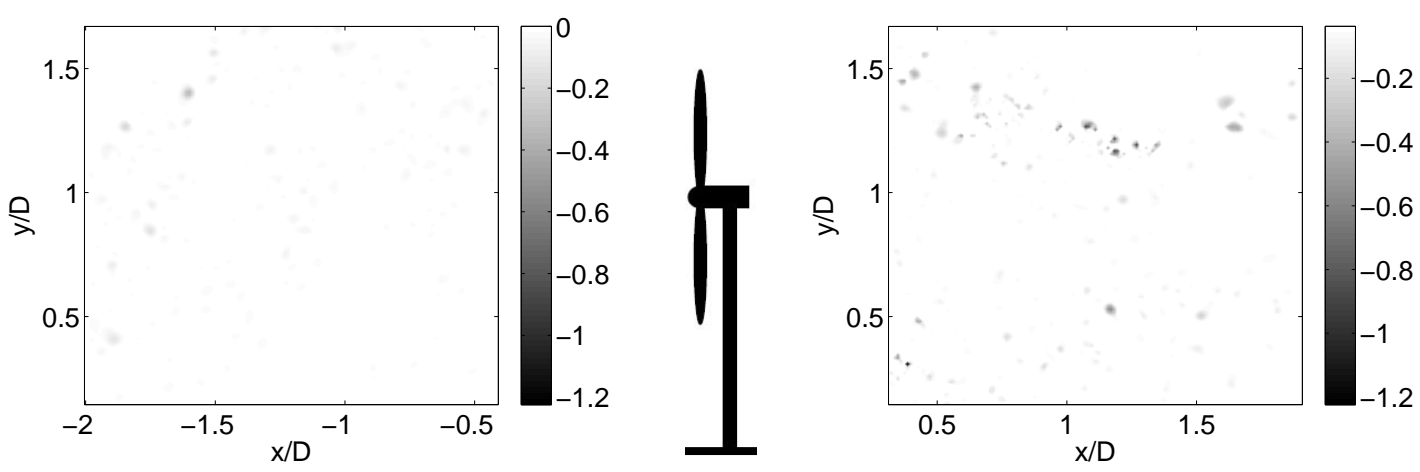

Figure D1: $\lambda_{2}$-criterion, Frame 118 . 